\title{
(p)ppGpp inhibits 70S ribosome formation in Staphylococcus aureus by impeding GTPase-ribosome interactions
}

\author{
Daniel J. Bennison ${ }^{1,4}$, Jose A. Nakamoto ${ }^{2}$, Timothy D. Craggs ${ }^{3}$, Pohl Milón ${ }^{2}$, John B. Rafferty ${ }^{4}$ and \\ Rebecca M. Corrigan ${ }^{1,4 *}$
}

\author{
${ }^{1}$ The Florey Institute, Department of Molecular Biology and Biotechnology, University of Sheffield, \\ Sheffield, S10 2TN, United Kingdom \\ 2Laboratory of Applied Biophysics and Biochemistry, Centre for Research and Innovation, Health \\ Sciences Faculty, Universidad Peruana de Ciencias Aplicadas (UPC), Lima, Peru \\ ${ }^{3}$ Sheffield Institute for Nucleic Acids, Department of Chemistry, University of Sheffield, Sheffield, S10 \\ 2TN, United Kingdom \\ ${ }^{4}$ Department of Molecular Biology and Biotechnology, University of Sheffield, Sheffield, S10 2TN, \\ United Kingdom. \\ * Correspondence: Tel +44 114222 4238; Email r.corrigan@sheffield.ac.uk
}

\begin{abstract}
During nutrient limitation, bacteria produce the alarmones ( $\mathrm{p}$ )ppGpp as effectors of the stress signalling network termed the stringent response. Screening for ( $\mathrm{p}$ )ppGpp-binding targets within Staphylococcus aureus identified four ribosome-associated GTPases (RA-GTPases), RsgA, RbgA, Era and HfIX, each of which are cofactors in ribosome assembly, where they cycle between the ON (GTP-bound) and OFF (GDP-bound) states. Entry into the OFF-state from the ON-state occurs upon hydrolysis of GTP, with GTPase activity increasing substantially upon ribosome association. When bound to (p)ppGpp, GTPase activity is inhibited, reducing $70 S$ ribosome assembly. Here, we sought to determine how (p)ppGpp impacts RA-GTPase-ribosome interactions by examining the affinity and kinetics of binding between RA-GTPases and ribosomes in various nucleotide-bound states. We show that RA-GTPases preferentially bind to 5'-diphosphate-containing nucleotides GDP and ppGpp over GTP, which is likely exploited as a regulatory mechanism within the cell. Binding to (p)ppGpp reduces stable association of RA-GTPases to ribosomal subunits compared to the GTP-bound state both in vitro and within bacterial cells by inducing the OFF-state conformation. We propose that in this conformation, the G2/switch I loop adopts a conformation incompatible with ribosome association. Altogether, we highlight (p)ppGppmediated inhibition of RA-GTPases as a major mechanism of stringent response-mediated growth control.
\end{abstract}




\section{INTRODUCTION}

The prokaryotic $70 \mathrm{~S}$ ribosome is an essential and complex macromolecular assembly responsible for the translation of messenger RNA (mRNA) into functional proteins. It comprises a large $50 \mathrm{~S}$ and a small 30 S subunit, which consist of 33 ribosomal proteins (r-proteins: L1-L36) associated with two ribosomal RNAs (rRNA), and 21 r-proteins (S1-S21) with one rRNA, respectively. Due to the energetic cost of ribosome synthesis and the intricacy of assembly, cofactors play a vital role in ensuring the correct conformation of the complete $70 S$ (1). One class of assembly cofactors are the ribosome-associated GTPases (RA-GTPases), a subset of P-loop GTPases within the Translation Factor Associated (TRAFAC) family, of which the proteins RsgA, RbgA, Era and HfIX are members. RA-GTPases have a highly conserved G-domain housing the catalytic G1-G5 motifs (Supplementary Figure S1), flanked by one or more highly variable accessory domains that convey targeting and additional functionality to the enzymes (Figure 1A) (2-6). The high degree of sequence identity (Supplementary Figure S1A) and structural conservation (Supplementary Figure S1B-E) between functional motifs within the nucleotidebinding pocket suggests a consistent mechanism of binding among these P-loop RA-GTPases.

Due to the variation in accessory domains, each RA-GTPase associates with a distinct area of the ribosome to coordinate a maturation event. Cycling between the GTP-bound ON and GDP-bound OFF states enables these proteins to act as molecular checkpoints of ribosome assembly by monitoring the maturation state of individual subunits (7). Although it is unclear what the precise roles of RAGTPases are in ribosomal maturation, they have been suggested to sterically prevent the premature association of other r-proteins (8). Unknown maturation events then act as activators of GTPase activity, enabling entry into the GDP-bound OFF state and subsequent dissociation from the ribosome (7). In addition to regulating the recruitment of r-proteins, RA-GTPases have been postulated to recruit RNA processing enzymes directly. For instance, the RA-GTPase Era can interact with several proteins involved in 16S rRNA maturation, including YbeY, an endonuclease involved in 16S processing in Escherichia coli (9), and CshA, a DEAD-box RNA helicase (10), pointing to a role for this group of enzymes as hub proteins that facilitate maturation events.

During periods of starvation, bacteria produce the alarmones guanosine penta- and tetraphosphate (collectively referred to as (p)ppGpp), which function as the mediators of a stress signalling system termed the stringent response (11). Amidst this response, the concentration of (p)ppGpp within the cell can reach between 1 - $2 \mathrm{mM}$ with a concurrent drop in GTP levels $(12,13)$. This results in a plethora of downstream effects, including alterations to transcription, translation and DNA replication, as well as regulating late-stage growth phases such as sporulation or biofilm formation (1416). Our previous work identified the four RA-GTPases (RsgA, RbgA, Era and HfIX: Figure $1 A$ ) in the pathogenic bacterium Staphylococcus aureus as enzymes that can bind to and are inhibited by (p)ppGpp, resulting in a negative impact on 70 S ribosome assembly and growth (17). RsgA is a nonessential, highly conserved late-stage $30 \mathrm{~S}$ assembly cofactor $(17,18)$, that has been implicated in the docking of helix 44 (h44) of the 16S rRNA into the correct conformation and therefore correct maturation of the decoding centre prior to subunit joining $(4,19)$. Era is a highly conserved protein, known to interact with the $3^{\prime}$ end of the pre-16S rRNA (3) where it monitors the ribonuclease processing 
state of this region. Furthermore, since Era docking occurs at the same site as r-protein S1 adjacent to the anti-Shine-Dalgarno sequence, it can also sterically occlude initiation factor 3 (IF3) binding and hence prevent formation of the $30 \mathrm{~S}$ pre-initiation complex (20). RbgA is a late-stage $50 \mathrm{~S}$ binding protein, implicated in RNA binding and remodelling $(6,21)$. Finally, $\mathrm{HfIX}$ is a $30 \mathrm{~S}, 50 \mathrm{~S}$ and $70 \mathrm{~S}$ binding protein that has been implicated in the splitting and subsequent repair of heat-stalled $70 \mathrm{~S}$ ribosomes (22). $\mathrm{HfIX}$ also contributes directly to $70 \mathrm{~S}$ levels through GTPase-dependent splitting of the $100 \mathrm{~S}$ hibernation complex to enable rapid recovery of active $70 S$ ribosomes when cellular energy levels rise, a process that is inhibited when bound to (p)ppGpp (23).

The binding of pppGpp to RbgA has previously been suggested to enhance the affinity of this protein for the mature $50 S$ subunit when compared to the GTP-bound form (24). More recently the crystal structure of $S$. aureus RbgA in complex with both ppGpp and pppGpp was solved, revealing a competitive mode of inhibition at the catalytic centre (6). These findings have led to a proposed model where RbgA-(p)ppGpp likely sequesters $50 \mathrm{~S}$ ribosomal subunits to prevent formation of active $70 \mathrm{~S}$ ribosomes (6). Here, we further characterise the four RA-GTPases RsgA, RbgA, Era and HflX, to investigate the relationship between RA-GTPases and stringent response-mediated control of ribosome assembly in S. aureus. We find that the $5^{\prime}$ diphosphate nucleotides GDP and ppGpp can bind to these enzymes with higher affinity than the $5^{\prime}$ triphosphate-containing GTP or pppGpp, suggesting that occupancy of the binding site is strongly dependent on a cellular excess of GTP over GDP, which occurs in exponential and non-stressed cells (25). Contrary to previous models, we demonstrate that interactions with (p)ppGpp prevent the association of RA-GTPases to the ribosome, both in vitro and in $S$. aureus. To examine mechanistically how (p)ppGpp can prevent RA-GTPase-ribosome interactions we use X-ray crystallography, revealing that (p)ppGpp binding causes the RA-GTPases to adopt a conformation similar to the inactive GDP-bound OFF state, with the switch I/G2 loop required for GTP hydrolysis extended away from the catalytic site where it could sterically hinder interactions with the ribosome. Altogether, we propose a mechanism behind ( $\mathrm{p}$ )ppGpp-controlled inhibition of ribosome assembly and increase our understanding of stringent response-mediated translational control by means of RA-GTPase inhibition.

\section{MATERIALS AND METHODS}

\section{Bacterial strains and culture conditions}

E. coli strains were grown in Luria Bertani broth (LB) and S. aureus strains in tryptic soy broth (TSB) at $37^{\circ} \mathrm{C}$ or $30^{\circ} \mathrm{C}$ with aeration. Strains and primers are listed in Supplementary Tables S1 and S2. Antibiotics were used when appropriate at the following concentrations: kanamycin $30 \mu \mathrm{g} / \mathrm{ml}$, carbenicillin $50 \mu \mathrm{g} / \mathrm{ml}$, spectinomycin $250 \mu \mathrm{g} / \mathrm{ml}$ and tetracycline $2 \mu \mathrm{g} / \mathrm{ml}$. pET28b-era G2 was created using overlap primer PCR with plasmid pET28b-era as a template and primers as listed in Supplementary Table S2. pCN55iTET-era-His was constructed by amplifying era using primers RMC157/RMC536 from LAC* genomic DNA and cloning into the Kpnl/Sacl sites of pCN55iTET. All plasmids were initially transformed into $E$. coli strain XL1-Blue and sequences of all inserts were verified 
by fluorescence automated sequencing by GATC. For protein expression and purification, all pET28b derived plasmids were transformed into E. coli strain BL21 (DE3). All S. aureus plasmids were first electroporated into RN4220 $\Delta s p a$, before isolation and electroporation into LAC* $\Delta$ era.

\section{GTPase assays}

GTPase activity assays were performed as previously described (10). Briefly, the ability of proteins to hydrolyse GTP was determined by incubating $100 \mathrm{nM}$ recombinant protein with $100 \mathrm{nM}$ S. aureus $70 \mathrm{~S}$ ribosomes, $1 \mu \mathrm{M}$ GTP and $2.78 \mathrm{nM} \mathrm{\alpha -}{ }^{32} \mathrm{P}-\mathrm{GTP}$ in $40 \mathrm{mM}$ Tris $\mathrm{pH}$ 7.5, $100 \mathrm{mM} \mathrm{NaCl}(100 \mathrm{mM} \mathrm{KCl}$ for $\mathrm{RbgA}$ ), $10 \mathrm{mM} \mathrm{MgCl}$ at $37^{\circ} \mathrm{C}$ for the indicated times. All reactions were also set up in the absence of enzymes to monitor spontaneous GTP hydrolysis. Reactions were heat inactivated at $95^{\circ} \mathrm{C}$ for 5 mins to precipitate proteins and release bound nucleotide. Precipitated proteins were pelleted by centrifugation at $17,000 \times \mathrm{g}$ for $10 \mathrm{~min}$. Reaction products were visualized by thin layer chromatography (TLC) in PEl cellulose TLC plates (Macherey-Nagel) and separated using $0.75 \mathrm{M} \mathrm{KH}_{2} \mathrm{PO}_{4}, \mathrm{pH} 3.6$ buffer. The radioactive spots were exposed to a BAS-MS Imaging Plate (Fujifilm), visualised using an LA 7000 Typhoon Phosphorlmager (GE Healthcare), and images quantified using ImageQuant (GE Healthcare).

\section{Synthesis of ${ }^{32} \mathrm{P}-(\mathrm{p}) \mathrm{ppGpp}$, differential radial capillary action of ligand assays (DRaCALA)}

The synthesis of (p)ppGpp and DRaCALA binding and competition assays were performed as described previously (17).

\section{Protein purifications}

Proteins were purified from 1-2 L E. coli BL21 DE3 cultures. Cultures were grown at $37^{\circ} \mathrm{C}$ to an $\mathrm{OD}_{600}$ of 0.5-0.7, expression was induced with $1 \mathrm{mM}$ isopropyl $\beta$-D-1-thiogalactopyranoside (IPTG) and incubated for $3 \mathrm{~h}$ at $30^{\circ} \mathrm{C}$. Cell pellets were resuspended in $5 \mathrm{ml}$ Buffer A (50 mM Tris pH 7.5, $150 \mathrm{mM}$ $\mathrm{NaCl}, 5 \%$ glycerol, $10 \mathrm{mM}$ imidazole) and lysed by sonication upon addition of $20 \mu \mathrm{g} / \mathrm{ml}$ lysozyme and $30 \mu \mathrm{g} / \mathrm{ml}$ RNase A. Protein purifications were performed by nickel affinity chromatography. The filtered cell lysate was loaded onto a $1 \mathrm{ml}$ HisTrap HP Ni${ }^{2+}$ column (GE Healthcare) before elution using a gradient of Buffer B (50 mM Tris pH 7.5, $200 \mathrm{mM} \mathrm{NaCl}, 5 \%$ glycerol, $500 \mathrm{mM}$ imidazole). Protein containing fractions were dialysed in $50 \mathrm{mM}$ Tris- $\mathrm{HCl} \mathrm{pH} \mathrm{7.5,} 200 \mathrm{mM} \mathrm{NaCl}, 5 \%$ glycerol before concentration using a $10 \mathrm{kDa}$ centrifugal filter (Thermo Scientific) and storage at $-80^{\circ} \mathrm{C}$. Protein for use in crystallography was dialysed into $25 \mathrm{mM}$ Tris- $\mathrm{HCl} \mathrm{pH} \mathrm{7.5,200} \mathrm{mM} \mathrm{NaCl}$ and used immediately. Protein concentrations were determined by absorbance at $280 \mathrm{~nm}$ using appropriate extinction coefficients. A $260 / 280_{2}$ ratios were monitored to ensure preparations had low RNA/nucleotide contamination $(<5 \%)$, indicated by a ratio below 0.8 . The extinction coefficients at $280 \mathrm{~nm}$ for each protein and their mutant variants were calculated from the primary structure: Era: $25900 \mathrm{M}^{-1} \mathrm{~cm}^{-1}$, RsgA: 
$23505 \mathrm{M}^{-1} \mathrm{~cm}^{-1}$, RbgA: $40910 \mathrm{M}^{-1} \mathrm{~cm}^{-1}, \mathrm{HflX}: 24870 \mathrm{M}^{-1} \mathrm{~cm}^{-1}$. Typically, protein purity was above $95 \%$ as assayed by $12 \%$ SDS-PAGE and Coomassie-blue staining.

\section{$30 S, 50 S$ and $70 S$ ribosome purification}

705 ribosomes were purified as described (17), with the following exceptions: following purification of mature $70 \mathrm{~S}$ ribosomes, the ribosome pellet was resuspended in dissociation buffer $(20 \mathrm{mM}$ Tris $\mathrm{pH} 7.5$, $120 \mathrm{mM} \mathrm{NH}_{4} \mathrm{Cl}, 1.5 \mathrm{mM} \mathrm{MgCl}_{2}$ and $2 \mathrm{mM} \beta$-mercaptoethanol), and quantified using the absorbance at $260 \mathrm{~nm}$ as described (26). 50 A260 units of $70 \mathrm{~S}$ ribosomes were applied to a $10-40 \%$ continuous sucrose gradient made up in dissociation buffer and separated at $111,000 \times \mathrm{g}$ for 16 hours. Gradients were fractionated by upwards displacement of $250 \mu$ laliquots, which were analysed for RNA content at an absorbance of $260 \mathrm{~nm}$. Fractions containing $30 \mathrm{~S}$ and $50 \mathrm{~S}$ ribosomal subunits were pooled separately, and purification was continued as described (26).

\section{In vitro ribosome association assays}

$500 \mathrm{nM}$ recombinant 6xHis-tagged RA-GTPase was incubated at room temperature for 5 mins with 200 $\mathrm{nM}$ S. aureus 70 ribosomes in dissociation buffer $\left(20 \mathrm{mM}\right.$ Tris $\mathrm{pH} 7.5,120 \mathrm{mM} \mathrm{NH}_{4} \mathrm{Cl}, 1.5 \mathrm{mM} \mathrm{MgCl}_{2}$ and $2 \mathrm{mM} \beta$-mercaptoethanol) in the apo form and in the presence of $40 \mu \mathrm{M}$ GTP, GMPPNP, GDP, ppGpp or pppGpp. The resultant reaction $(150 \mu \mathrm{l})$ was layered onto a $10-40 \%$ continuous sucrose density gradient in dissociation buffer. Subsequently, gradients were centrifuged for $16 \mathrm{~h}$ at $111,000 \mathrm{x}$ $\mathrm{g}$ in order to separate the $30 \mathrm{~S}$ and $50 \mathrm{~S}$ subunits. Gradients were fractionated by upwards displacement of $250 \mu \mathrm{l}$ aliquots, which were analysed for RNA content at an absorbance of $260 \mathrm{~nm}$. Fractions containing $30 \mathrm{~S}$ and $50 \mathrm{~S}$ ribosomal subunits were pooled separately and the protein content was precipitated by the addition of $10 \% \mathrm{v} / \mathrm{v}$ trichloroacetic acid (TCA) and incubation for $3 \mathrm{~h}$ at $4^{\circ} \mathrm{C}$. Samples were centrifuged at $17,000 \times \mathrm{g}$ for 5 mins and washed twice with ice-cold acetone prior to drying of the pellets at $37^{\circ} \mathrm{C}$ for 10 mins. Pellets were resuspended in $2 x$ SDS-PAGE sample buffer (62.5 mM Tris$\mathrm{HCl} \mathrm{pH} 6.8,2 \%$ SDS, $10 \%$ glycerol, $0.01 \%$ bromophenol blue, $10 \% \mathrm{v} / \mathrm{v} \beta$-mercaptoethanol), proteins were separated using a $10 \%$ SDS-PAGE gel and transferred onto a PVDF Immobilon-P membrane (Merck Millipore). The membrane was blocked with 5\% w/v milk in TBST (50 mM Tris-HCl pH 7.6, 150 $\mathrm{mM} \mathrm{NaCl}, 0.1 \%$ Tween 20), probed using 1:500 monoclonal anti-His HRP-conjugated antibodies (Sigma) and imaged using a ChemiDoc MP (Bio-Rad). Band densitometry was performed using ImageJ.

\section{Growth and in vivo ribosome association assays}

S. aureus strains were grown overnight in TSB containing the appropriate antibiotics. Overnight cultures were diluted to a starting $\mathrm{OD}_{600}$ of $0.05 \mathrm{in}$ the presence of $100 \mathrm{ng} / \mathrm{ml}$ Atet and appropriate antibiotics and 
grown at $37^{\circ} \mathrm{C}$ with aeration, with $\mathrm{OD}_{600}$ values determined at $2 \mathrm{~h}$ intervals. For ribosome association assays, a culture of LAC* $\triangle$ era pCN55iTET-era-his was split at an $\mathrm{OD}_{600}$ of 0.6 and fractions were either left uninduced or were induced with either 0.05 or $60 \mu \mathrm{g} / \mathrm{ml}$ mupirocin at $37^{\circ} \mathrm{C}$ for 30 mins. After growth, all cultures were incubated with $100 \mu \mathrm{g} / \mathrm{ml}$ chloramphenicol at $37^{\circ} \mathrm{C}$ for $3 \mathrm{mins}$, then cooled to $4^{\circ} \mathrm{C}$. Cells were centrifuged at $4,000 \times \mathrm{g}$ for 10 mins and pellets resuspended to an $\mathrm{OD}_{600}$ of 35 in dissociation buffer (20 mM Tris pH 7.5, $120 \mathrm{mM} \mathrm{NH}_{4} \mathrm{Cl}, 1.5 \mathrm{mM} \mathrm{MgCl}_{2}$ and $2 \mathrm{mM} \beta$-mercaptoethanol). Cells were lysed through the addition of $0.5 \mu \mathrm{g} / \mathrm{ml}$ lysostaphin and $75 \mathrm{ng} / \mathrm{ml}$ DNase for $60 \mathrm{mins}$ at $37^{\circ} \mathrm{C}$. Lysates were centrifuged at $17,000 \mathrm{xg}$ for $10 \mathrm{~min}$ to remove cell debris and $250 \mu \mathrm{l}$ of the lysate was layered onto a $10-40 \%$ continuous sucrose gradient in dissociation buffer. Subunit separation was continued as per the in vitro method and associated C-terminally histidine-tagged Era (Era-His) was quantified via western blotting and band densitometry (ImageJ). Crude lysates were loaded alongside pulled-down protein to verify Era-His expression level. Staining of the blotting membrane with Ponceau S in 5\% acetic acid was used to ensure consistent lysate loading prior to membrane blocking. Membranes were incubated with staining solution for up to 5 minutes and washed with distilled water until the background was clear. Following imaging, the Ponceau $S$ was removed by repeated wash steps using PBS.

\section{Ribosome profiles from $S$. aureus cell extracts}

Crude isolations of ribosomes from S. aureus cell extracts were achieved as described by Loh et al. with some modifications (27). Briefly, $100 \mathrm{ml}$ cultures of the different $S$. aureus strains were grown to an $\mathrm{OD}_{600}$ of 0.4 in TSB medium with $100 \mathrm{ng} / \mathrm{ml}$ anhydrotetracycline (Atet). $100 \mu \mathrm{g} / \mathrm{ml}$ chloramphenicol was added to each culture and incubated for $3 \mathrm{~min}$ before being cooled to $4^{\circ} \mathrm{C}$ to enhance the pool of $70 \mathrm{~S}$ ribosomes. Pelleted cells were suspended in association buffer $(20 \mathrm{mM}$ Tris-HCl pH 7.5, $8 \mathrm{mM}$ $\mathrm{MgCl}_{2}, 30 \mathrm{mM} \mathrm{NH}_{4} \mathrm{Cl}$ and $2 \mathrm{mM} \beta$-mercaptoethanol) and normalized to an $\mathrm{OD}_{600}$ of 15 . Cells were lysed by the addition of $0.2 \mu \mathrm{g} / \mathrm{ml}$ lysostaphin and $75 \mathrm{ng} / \mathrm{ml}$ DNase and incubated for $60 \mathrm{~min}$ at $37^{\circ} \mathrm{C}$. Cell debris was removed by centrifugation at $17,000 \mathrm{xg}$ for $10 \mathrm{~min}$. Clarified lysates $(250 \mu \mathrm{l})$ were layered onto $10-50 \%$ discontinuous sucrose density gradients made in association buffer. Gradients were centrifuged for $7 \mathrm{~h}$ at $192,100 \times \mathrm{g}$. Gradients were fractionated by upwards displacement of $250 \mu \mathrm{l}$ aliquots, which were analysed for RNA content by absorbance at $260 \mathrm{~nm}$.

\section{Crystallisation of RsgA}

The purified recombinant protein consisted of 311 residues, comprising 291 residues of S. aureus RsgA with an N-terminal 20 residue tag MGSSHHHHHHSSGLVPRGSH. It was simultaneously buffer exchanged into $25 \mathrm{mM}$ Tris- $\mathrm{HCl} \mathrm{pH} 7.5,200 \mathrm{mM} \mathrm{NaCl}$ buffer and concentrated to $30 \mathrm{mg} / \mathrm{ml}$ for crystallization screening using the sitting drop vapour diffusion method. Each droplet contained $200 \mathrm{nl}$ protein solution and $200 \mathrm{nl}$ crystallisation reagent from an adjacent well of $50 \mu \mathrm{l}$ volume. Figures were prepared in Pymol (The PyMOL Molecular Graphics System, Version 2.0 Schrödinger, LLC), with the exception of electron density maps which were generated using COOT $(28,29)$. 


\section{RsgA-ppGpp}

The concentrated RsgA solution was supplemented with $2 \mathrm{mM} \mathrm{MgCl}_{2}$ and $2 \mathrm{mM} \mathrm{ppGpp.} \mathrm{Successful}$ crystallisation was observed when this sample was mixed $1: 1$ with well solution containing $0.2 \mathrm{M}$ sodium citrate tribasic dihydrate, $0.1 \mathrm{M}$ Bis-Tris propane $\mathrm{pH} 6.5$ and 20\% (w/v) PEG 3350, and incubated at $17^{\circ} \mathrm{C}$. Rod shaped crystal clusters appeared after a few days. Crystals were transferred to a cryoprotectant solution consisting of mother liquor with $15 \%$ ethylene glycol added and flash cooled in liquid $\mathrm{N}_{2}$. X-ray diffraction data were collected from a single crystal on beamline i04 at the Diamond Light Source national synchrotron facility at a wavelength of $0.97949 \AA$. The ppGpp-bound crystals diffracted to a resolution of $1.94 \AA$ (PDB: $6 \mathrm{ZHL}$ ). Initial processing was completed using the Xia2 pipeline (30). The crystals belonged to the space group P2 $12{ }_{1}{ }_{21}$ (Supplementary Table S3). The structure of RsgA-ppGpp was solved via molecular replacement, using the previously published Bacillus subtilis homologue YloQ (PDB: $1 \mathrm{T9H}$ ) as a model. The structure contained one RsgA monomer in the asymmetric unit. Molecular replacement was carried out using Phaser from within the CCP4 suite $(31,32)$. The structure was refined via rounds of manual model building and refinement using COOT (29) and REFMAC5 (33). The final model was validated using MOLPROBITY (34). Residues 181-200 were lacking electron density and as such were omitted from the final model.

\section{Apo RsgA}

Crystallisation of apo RsgA was achieved when the concentrated protein sample was mixed 1:1 with well solution containing $0.15 \mathrm{M}$ ammonium sulphate, $0.1 \mathrm{M}$ MES pH 6.0 and 15\% (w/v) PEG 4000 and incubated at $17^{\circ} \mathrm{C}$. A single rod shaped crystal formed after a few weeks and diffracted to $2.01 \AA$ resolution (PDB: 6ZJO). Initial processing was completed using the Xia2 pipeline and the crystal belonged to the space group P1211 (Supplementary Table S3). The structure was solved via molecular replacement as above using the available RsgA-ppGpp structure as a model with ligands removed and contained two RsgA monomers in the asymmetric unit. Iterative rounds of modelling, refinement and validation were carried out as above. Residues 180-200 (Chain A) and 179-200 (Chain B) were lacking electron density and as such omitted from the model.

\section{Fluorescent labelling of proteins}

$200 \mu \mathrm{M}$ recombinant protein was incubated with $5 \mathrm{mM}$ dithiothreitol (DTT) for $1 \mathrm{~h}$ at room temperature. DTT was removed via two consecutive passes through a PD-10 Sephadex G-25 M buffer exchange column (GE Healthcare) as per the manufacturer's instructions into labelling buffer ( $50 \mathrm{mM} \mathrm{HEPES} \mathrm{pH}$ $7.1,200 \mathrm{mM} \mathrm{KCl}, 5 \%$ glycerol, $120 \mu \mathrm{M}$ TCEP). Flow-through was analysed for protein content at 280 $\mathrm{nm} .50 \mu \mathrm{M}$ of reduced protein was incubated with $100 \mu \mathrm{M}$ ATTO 488-maleimide (ATTO-TEC) overnight at $4^{\circ} \mathrm{C}$, shielded from light and subject to gentle shaking. The reaction was stopped by addition of $6 \mathrm{mM}$ $\beta$-mercaptoethanol and mixtures were applied to a $1 \mathrm{ml} \mathrm{HisTrap} \mathrm{HP} \mathrm{Ni}{ }^{2+}$ column (GE Healthcare) before elution using a gradient of Buffer $\mathrm{B}(50 \mathrm{mM}$ Tris pH 7.5, $200 \mathrm{mM} \mathrm{NaCl}, 5 \%$ glycerol, $500 \mathrm{mM}$ imidazole) 
and subsequent dialysis to remove imidazole. Labelling efficiency was calculated in accordance with the fluorescent dye manufacturer's guidelines.

\section{Stopped-flow fluorescence kinetics measuring ribosome association}

For initial controls, $0.2 \mu \mathrm{M}$ Atto-488-labelled proteins were rapidly mixed with $0.2 \mu \mathrm{M} \mathrm{E}$. coli $50 \mathrm{~S}$ ribosomal subunits (purified as described elsewhere (35)) in TAKM7 buffer (25 mM Tris-HCl pH 7.4, 70 $\mathrm{mM}$ ammonium acetate, $30 \mathrm{mM} \mathrm{KCl}$ and $7 \mathrm{mM} \mathrm{MgCl}_{2}$ ) using an SX20 stopped-flow apparatus (Applied Photophysics) in the presence or absence of GTP, ppGpp and pppGpp. Equal volumes (60 $\mu$ l) of each reactant were rapidly mixed at $25^{\circ} \mathrm{C}$. Atto- 488 was excited using a $470 \mathrm{~nm}$ LED and fluorescence was detected through a $515 \mathrm{~nm}$ long-pass filter. Reactions were monitored for 10 seconds using a logarithmic sampling method, with 1000 total datapoints per reaction. Each condition was subject to at least 5 technical repeats, with curves representing the mean average fluorescence of the technical repeats.

For titrations, $0.075 \mu \mathrm{M}$ RbgA or $0.05 \mu \mathrm{M} \mathrm{HflX} \mathrm{labelled} \mathrm{proteins} \mathrm{were} \mathrm{mixed} \mathrm{with} \mathrm{a} 200$-fold excess of GTP, ppGpp or pppGpp (15 $\mu \mathrm{M}$ for RbgA and $10 \mu \mathrm{M}$ for HfIX) in TAKM7 buffer just prior to use. E. coli ribosomal $50 \mathrm{~S}$ subunits were used in excess relative to the labelled protein in the presence of nucleotides, up to $0.8 \mu \mathrm{M}$. Samples were then loaded separately into an SX20 stopped flow apparatus. Equal volumes $(60 \mu \mathrm{l})$ of each reactant were rapidly mixed at $25^{\circ} \mathrm{C}$ and fluorescence emission was monitored as described above. The resultant fluorescence timecourses were fitted using the double exponential function $F=F_{0}+\left(A_{1} \times e^{-k a p p_{1} t}\right)+\left(A_{2} \times e^{-k a p p_{2} t}\right)$ with fluorescence signal at time $t(F)$, initial fluorescence signal $\left(F_{0}\right)$, the amplitude of signal change of the first exponential $\left(A_{1}\right)$,

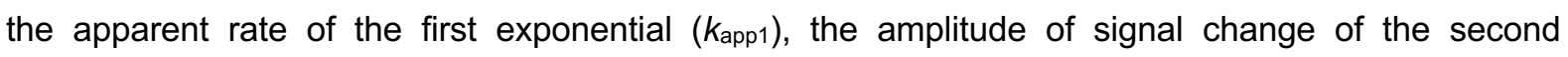
exponential $\left(A_{2}\right)$, the apparent rate of the second exponential $\left(k_{\text {app } 2}\right)$ and time $(t)$. Each timecourse was fitted individually, with curves shown representing the mean average of at least 5 technical replicates. If necessary, a linear term was included. Data was normalised to the mean of the first 10 fluorescence measurements. The microscopic constants $k_{1}, k_{-1}, k_{2}$ and $k_{-2}$ were calculated by plotting both the sum and product of the apparent rates $k_{\text {app } 1}$ and $k_{\text {app } 2}$ for each titration and analysing the resulting linear relationship using linear regression. Briefly, taking $A$ as the linear regression of the sum of $k_{a p p 1}$ and $k_{\text {app2, and } B}$ as the linear regression of the product of $k_{\text {app } 1}$ and $k_{\text {app2 }}$, kinetic parameters were determined as follows: $k_{1}=\operatorname{slope}(A), k_{-1}=\operatorname{intercept}(A)-\frac{\operatorname{slope}(B)}{\operatorname{slope}(A)}, k_{2}=\operatorname{intercept}(A)-k_{-1}-k_{-2}$, and $k_{-2}=$ $\frac{\operatorname{intercept}(B)}{k_{-1}}$. Dissociation constants $\left(K_{d}\right)$ were calculated using the following equation: $K_{d}=\frac{\left(\frac{k_{-1} \times k_{-2}}{k_{1}}\right)}{\left(k_{-2}+k_{2}\right)}$.

\section{Statistics}

Statistical analyses were performed using Graphpad Prism 8.0 software. Statistical differences between samples were assessed using one-way analysis of variance (ANOVA), followed by Tukey's multiple comparisons test. 


\section{RESULTS}

\section{RA-GTPases preferentially bind 5' diphosphate-containing nucleotides GDP and ppGpp}

The RA-GTPases RsgA, Era, RbgA and HflX can bind to the guanosine nucleotides GTP, GDP, ppGpp and pppGpp. Our previous work observed higher binding affinities for ppGpp over GTP, pointing towards a difference in binding between $5^{\prime}$ di- or triphosphate nucleotides (17). To examine the nucleotide binding affinities of these RA-GTPases for GDP in comparison to ppGpp, pppGpp and GTP, we used a differential radial capillary action of ligand assay (DRaCALA) (Figure 1B, Supplementary Figure S2A-C, (36)). In each case, the affinities of $5^{\prime}$ diphosphate-containing GDP and ppGpp were similar in the low $\mu \mathrm{M}$ range and were 2-6-fold higher than the affinities of either GTP or pppGpp (Supplementary Table S4). This supports a previous observation that ppGpp is a more potent inhibitor of GTPase activity than pppGpp (17).

Structural data places (p)ppGpp within the GTP-binding site of the RA-GTPase RbgA (6). Based on our measured affinities (Supplementary Table S4), we speculate that both GDP and ppGpp will out-compete other nucleotides for occupancy of the binding site. To examine this, competition assays were performed in which the binding of a radiolabelled nucleotide was challenged with an excess of unlabelled nucleotides (Figure 1C, Supplementary Figure S2D-F). In each case, addition of cold unlabelled nucleotide reduced occupancy of the labelled nucleotide, with the exception of the ATP control. This is likely due to the much lower affinity of RA-GTPases for adenosine bases conveyed by a contact from the conserved aspartate residue of the $\mathrm{G} 4$ motif to the 2-amino group of the guanosine base (37). A hierarchy of binding could be established depending on the level of competition provided by each unlabelled nucleotide, with GDP and ppGpp competing more effectively (Figure 1C, Supplementary Figure S2D-F). This suggests that GTP occupancy, and hence activity, of these RAGTPases is strongly dependent on the cellular excess of GTP over GDP and ppGpp, which occurs during exponential growth when ribosomal biogenesis is at its peak (25). This ratio changes during stationary phase and upon induction of the stringent response, when cellular GTP levels decrease with a concurrent rise in (p)ppGpp $(12,38)$, shifting binding to favour a ppGpp-bound state. The greater affinity of these RA-GTPases to diphosphate-containing nucleotides would hence aid a rapid transition between the GTP-bound and ppGpp-bound states under conditions of stress.

\section{Interactions with (p)ppGpp reduce the affinity of RA-GTPases for the ribosome}

It is well characterised that rRNA transcription decreases during the stringent response (39). In addition, the GTPase activity of ribosome assembly cofactors is inhibited by ( $p$ )ppGpp, both of which contribute to a reduction in mature ribosomes within the cell (17). To examine mechanistically how (p)ppGppGTPase interactions affect the ability of RA-GTPases to associate with ribosomal subunits, we examined the association of each GTPase to either the $30 \mathrm{~S}$ or $50 \mathrm{~S}$ ribosomal subunit in the presence of GDP, GTP, ppGpp, pppGpp, as well as GMPPNP, a non-hydrolysable analogue of GTP. His-tagged GTPases were preincubated with highly pure, salt washed $70 S \mathrm{~S}$. aureus ribosomes in a low 
magnesium buffer to encourage ribosomal subunit dissociation, and the amount of each GTPase associated with each of the subunits was quantified by western immunoblot using anti-His antibodies after sucrose gradient separation (Figure 2). In all cases, we observed a marked decrease in association of each GTPase to the $30 \mathrm{~S}$ or 50 S subunits in the presence of GDP, ppGpp and pppGpp compared to the GMPPNP-bound state (Figure 2A-D). For Era and HflX, there was a similar level of subunit association when in the apo, GTP or GMPPNP-bound states, compared to a 2-fold reduction in ribosome binding when incubated with GDP, ppGpp or pppGpp (Figure 2C, 2D), suggesting that these GTPases can associate to the ribosome in the unbound state. The ability of Era to bind the $30 \mathrm{~S}$ in the absence of nucleotides has been reported previously, where it has been suggested that the apo form can bind to a secondary site $(3,40)$. The patterns exhibited by RsgA and RbgA were slightly different, with strong binding in the GMPPNP-bound state, whereas 3-6 fold weaker binding was observed in the apo, GTP, GDP, ppGpp and pppGpp-bound states (Figure 2A, 2B). It is worth noting that previous studies have suggested that the association of RbgA with the $50 \mathrm{~S}$ subunit is enhanced in the presence of pppGpp (24), a finding that is not replicated here. The apparent effect of ppGpp and pppGpp on ribosome association was comparable, which is not reflective of the differences in affinity (Figure 1B, Supplementary Figure S2A-C), although under the conditions tested here the excess of nucleotide would maintain an equilibrium favouring the nucleotide-bound state. Furthermore, the four RA-GTPases were found to be unable to hydrolyse pppGpp, and as such conversion of pppGpp to ppGpp was not responsible for the similar degree of inhibition of association. We postulate that the low level of binding observed when preincubated with GTP could be due to GTP hydrolysis during the 16 hour centrifugation step, likely causing the GTPases to enter the GDP-bound state and dissociate. This, in turn, may be enhanced by the higher affinity of GDP for these GTPases as compared to GTP (Supplementary Table S4). From these data, we show that GTP binding favours association of RsgA, $\mathrm{RbgA}$, Era and HfIX to ribosomal subunits, and that this interaction is inhibited when in the GDP-, ppGpp- or pppGpp-bound states.

\section{Binding kinetics of RA-GTPase-ribosome interactions}

To gain further insight into the binding mechanism and how (p)ppGpp reduces the association of RAGTPases to the ribosomal subunits, we used a stopped-flow technique with fluorescent derivatives of the RA-GTPases (Figure 3A). Structural predictions of all four R A-GTPases were built by homology modelling using available structures to assess the availability of suitable residues for fluorescence labelling (Supplementary Figure S3A, S3B)(41). Both RbgA and HflX were amenable to covalent linkage to the fluorophore Atto-488 using maleimide chemistry with exposed cysteine residues. RbgA contains one wild-type cysteine residue (C277) that is surface exposed in the $B$. subtilis crystal structure (PDB: 1PUJ) and is located towards the C-terminus of the protein (Supplementary Figure S3A). Based on the E. coli structure (PDB: 5ADY), HfIX contains two cysteines (Supplementary Figure S3B). C330 is predicted to be surface exposed and therefore amenable to labelling, while C45 is buried and is expected to show low accessibility for fluorescent labelling. Era, on the other hand, lacks any cysteine residues, while RsgA contains three conserved cysteine residues that coordinate the $\mathrm{Zn}^{2+}$ ion within the 
$\mathrm{Zn}^{2+}$-finger domain (ZNF), and as such both were not suitable for labelling. Both Atto488-labelled RbgA and $\mathrm{HflX}$ retained wild-type levels of GTPase activity, which can still be inhibited by ppGpp (Supplementary Figure S3C, S3D).

Using the fluorescent variants of RbgA and HfIX, we studied the binding mechanism of both to the 50 S ribosomal subunit in the GTP-, ppGpp- and pppGpp-bound states. First, the fluorescence change of each labelled protein was measured upon the interaction with activated mature ribosomal subunits in the presence of different nucleotides (Figure 3B, 3C). RbgA showed no change in fluorescence while in the apo state, indicating a lack of interaction with the ribosome. On the other hand, all nucleotide-bound states showed a large decrease in fluorescence when mixed with the 50 S subunit, consistent with some level of 50 S association taking place when bound to GTP, ppGpp or pppGpp (Figure 3B). HfIX, on the other hand, exhibited a fluorescence change upon mixing with the 50 S subunit in the absence or presence of all tested nucleotides (GTP, ppGpp and pppGpp), which could be taken as a direct measure of ribosome association changing the chemical environment of the fluorophore (Figure 3C).

Next, we used a constant concentration of protein in the presence of 200 -fold excess of each nucleotide and titrated it with increasing concentrations of ribosomal subunits (Supplementary Figure S4). Time traces appeared biphasic for both RA-GTPases independent of the nucleotide bound. Analysis of the fluorescent time traces with a double exponential equation yielded the apparent rates of association ( $k_{\text {app } 1, ~}$ app2) (Figure 3D, 3E, Supplementary Figure S5A, S5B), in accordance with a binding mechanism composed of two sequential steps. Thus, the mechanism describing the interaction $k_{1} \quad k_{2}$

(Equation 1: $P+S \rightleftharpoons P S^{\prime} \rightleftharpoons P S$ ) consists of an initial interaction and subsequent stabilisation of the $k_{-1} \quad k_{-2}$

factor on the ribosome, where $P$ is the protein, $S$ is the ribosomal subunit, $P S^{\prime}$ is the transient complex and $P S$ is the stable complex.

For two-step reactions, the apparent rate under conditions tested, $k_{a p p 1}$, is expected to increase linearly with increasing ligand concentration. On the other hand, $k_{\text {app } 2}$ is expected to align to a hyperbolic relationship as ligand concentration increases. This was the case for HflX complexed with GTP (Figure 3D, 3E). Thus, productive binding of the RA-GTPase appears to occur through two steps. When HfIX was incubated with ppGpp, the kapp1 increased linearly (Figure 3D), while $k_{\text {app2 }}$ did not depend on ribosome concentration (Figure $3 \mathrm{E}$ ), indicating that ppGpp hampers the accommodation step of the binding mechanism. On the other hand, if $\mathrm{HfIX}$ was complexed with pppGpp, neither $k_{\text {app }}$ depended on $50 \mathrm{~S}$ concentration, indicating that the alarmone drastically affects the mechanism of HfIX binding. In this case, the reaction appears rate-limited by an isomerization step of the RA-GTPase at $5 \mathrm{~s}^{-1}$ (Figure 3D). The linear increase in $k_{a p p 1}$ was 2 -fold greater for GTP than for ppGpp or pppGpp (Figure 3D), suggesting a greater rate of the fast-phase reaction. The kapp2 of the GTP-bound form showed a hyperbolic relationship tending to $2 \mathrm{~s}^{-1}$, while the linear relationship when bound to ppGpp was steady at $1.0 \mathrm{~s}^{-1}$ (Figure $3 \mathrm{E}$ ). This suggests that the second, slow-phase reaction is taking place while HfIX is bound to GTP but is reduced 4-fold when bound to ppGpp. Additionally, this suggests that one or more of the microscopic constants which contribute to the kapp2 in the two-step association reaction remains incomplete while in the ppGpp-bound state. 
Next, we used the sum and product of the $k_{\text {app } 1}$ and $k_{\text {app2 }}$ of each reaction (Figure $3 F, 3 G$ ) to estimate the microscopic constants defining the reaction for the GTP- and ppGpp-bound HfIX (Supplementary Table S5). ppGpp reduced the value of the initial binding constant $k_{1}$ by 2-3 fold, while drastically affecting $k_{2}$, indicating that the alarmone hampers proper accommodation of HfIX on the subunit (Figure 3F, 3G, Supplementary Table S5). On the contrary, the dissociation rate constants $k-1$ and $k_{-2}$ appeared less affected by ppGpp, remaining similar to those observed during the GTP-bound state (Supplementary Table S5). Altogether, our data indicates that (p)ppGpp induces a non-productive conformation of $\mathrm{HfIX}$, reducing the binding progression with the ribosomal subunit.

In the case of RbgA, all three tested nucleotides adhered to a two-step mechanism model, with $k_{\text {app } 1}$ increasing linearly with $50 S$ concentration, while $k_{\text {app2 }}$ appeared hyperbolic (Supplementary Figure S5A, S5B). Further analysis to estimate the microscopic constants indicated that (p)ppGpp increased the dissociation rate constant $k_{-1}$ by $3-5-$ fold as compared to GTP, whereas its association velocity $k_{1}$ appeared largely unaffected (Supplementary Table S5, Supplementary Figure S5C, S5D). Interestingly, ppGpp drastically reduced the accommodation rate constant $k_{2}$ similarly to $\mathrm{HflX}$, while pppGpp did not. Altogether, our results indicate that (p)ppGpp can program RbgA to adopt different conformations that ultimately reduce their binding affinity for the ribosome (Supplementary Table S5).

For both RA-GTPases, the $K_{d}$ of $50 S$ binding is lower in the GTP-bound state compared to the (p)ppGpp-bound state (Supplementary Table S5). It appears that the main difference on a kinetic level, in agreement with our previous observations regarding the accommodation step, is that the binding of (p)ppGpp prevents the initiation of the slow-phase reaction $\left(k_{2}\right)$. (p)ppGpp would therefore prevent the stable association of the RA-GTPase with the ribosomal subunit. The affinity is further affected by the increased $k_{-1}$ in the alarmone-bound states, which in addition to the lack of the second phase reaction while bound to (p)ppGpp, may lead to an increase in reversal reactions, enhancing the dissociation of the RA-GTPase from the ribosomal subunits. Altogether, the kinetic data are in accordance with the observation by western immunoblotting that RA-GTPases bind to target subunits more readily in the GTP-bound form but associate less readily in the presence of the stringent response alarmones (p)ppGpp. Specifically, (p)ppGpp appear to affect the forward reactions, consistent with inducing a nonproductive conformation of the RA-GTPases. This could lead to diminished association of RA-GTPases to ribosomes at physiologically relevant alarmone concentrations, impairing ribosome maturation under stress.

\section{Association of the RA-GTPase Era to the 30 S subunit decreases upon induction of the stringent response}

Upon induction of the stringent response, cellular levels of ( $p$ )ppGpp increase, while concentration of GTP drops (38). Having observed decreased association of RA-GTPases to ribosomal subunits in vitro, we wished to examine the interaction under more physiologically relevant conditions. To investigate RA-GTPases interactions with the ribosome in the bacterial cell, we used an era deletion mutant in the community-acquired methicillin-resistant $S$. aureus (CA-MRSA) strain $L A C^{*}$ that was available to us. This strain has a growth defect (Figure 4A) and has an abnormal cellular ribosomal profile when 
compared to the wild-type, with an accumulation of $50 \mathrm{~S}$ subunits and a loss of $70 \mathrm{~S}$ ribosomes (Figure $4 B, 4 C)(10,27,42)$, suggesting that the absence of this GTPase is preventing mature ribosome formation and growth. In order to establish whether induction of the stringent response in bacterial cells leads to a decrease in the association of Era to the $30 \mathrm{~S}$ subunit, the mutant was complemented with an anhydrotetracycline-inducible $6 x$ His-tagged version of era, yielding strain LAC* $\triangle$ era iTET-era-His. Having confirmed that the His-tagged version of the protein is expressed and restores the growth defect observed in era mutant strains (Figure 4A), we grew cells to exponential phase and induced the stringent response with mupirocin, an antibiotic that inhibits isoleucyl tRNA synthetase and is known to activate the stringent response in $S$. aureus (43). Cells were lysed and applied to $10-40 \%$ sucrose gradients in ribosome dissociation buffer for subunit separation via isopycnic ultracentrifugation. The $30 \mathrm{~S}$ pool was analysed for associated Era-His using $\alpha$-His western immunoblotting (Figure 4D). Crude lysates sampled prior to loading on the sucrose gradients were also analysed to ensure equal loading and equal expression of Era-His between samples (Supplementary Figure S6). In agreement with the in vitro data, the relative association of Era-His to the ribosome decreased at least 4-fold upon induction of the stringent response (Figure 4D). Altogether this in vitro and in vivo data support a model in which the stringent response impairs 70S ribosome assembly by disrupting the association of RA-GTPases with the immature ribosomal subunits, thus preventing correct ribosome maturation.

\section{Crystallisation of RsgA in the apo and ppGpp-bound states}

GTPases act as molecular switches, cycling between OFF (GDP-bound) and ON (GTP-bound) states. Structural studies of numerous GTPases have reported distinct conformations for both states, which are determined by the movement of the flexible switch I/G2 loop and the switch II/G3 loop (44). Often described as a loaded-spring mechanism, the conformational change occurs upon hydrolysis of GTP or the subsequent $\gamma$-phosphate release. Both switch I/G2 and switch II/G3 are responsible for coordinating the $\mathrm{Mg}^{2+}$ cofactor which interacts with the $\gamma$-phosphate of GTP via a conserved threonine residue in $\mathrm{G} 2$ and a glycine in G3. Upon hydrolysis of the $\gamma$-phosphate and $P_{i}$ dissociation, the protein relaxes into the OFF conformation.

To look more at the mechanism of ( $\mathrm{p}$ )ppGpp-mediated inhibition of RA-GTPases associating with ribosomal subunits, we solved the structures of RsgA in both the apo- (Figure 5A) and ppGppbound (Figure $5 \mathrm{~B}$ ) states by X-ray crystallography (Supplementary Table S3), in order to compare to already available GMPPNP- and GDP-bound structures. The $1.94 \AA$ structure of RsgA complexed with ppGpp reveals the presence of the nucleotide unambiguously represented in the electron density map (Supplementary Figure S7A), whereas the apo structure at $2.01 \AA$ is lacking any electron density in the nucleotide binding pocket. The overall structure of RsgA consists of three domains, the N-terminal OBfold, the central GTPase domain and a C-terminal ZNF (Figure 5A). Both the OB-fold and ZNF domains are involved in nucleotide recognition $(45,46)$, and target RsgA to the $30 \mathrm{~S}$ ribosomal subunit where they contact major helices of the $16 \mathrm{~S}$ rRNA (Figure 5C). The OB-fold is situated between h18 and h44, with the loop connecting $\beta_{1}$ and $\beta_{2}$ recognising the minor groove of h44 adjacent to the $30 \mathrm{~S}$ acceptor site 
(4). The ZNF contacts the $30 S$ head domain, making backbone contacts with $h 29$ and $h 30$, close to the interaction site of the P-site tRNA $(4,47)$. In E. coli RsgA (YjeQ), the G-domain also contacts h44 by means of a clamp adjacent to the interaction site of h45 and h24. This clamping interaction is facilitated by the $\beta_{6,7}$ hairpin and the switch I/G2 region (4), however this hairpin is lacking in $S$. aureus RsgA (Figure 5A, 5B), so it is likely that the G-domain interacts with h44 singly through the switch I/G2 region.

The ppGpp ligand is bound in an elongated conformation, where the $3^{\prime}$ and $5^{\prime}$-phosphate moieties face away from each other (Supplementary Figure S7A). The guanosine- 5 '-diphosphate backbone interacts with the G-domain in an identical manner to the more well-characterised GMPPNP (Supplementary Figure S7B, S7C) $(4,47)$, with the P-loop/G1 motif stabilising the $\alpha, \beta$-diphosphate and the G4 motif specifically recognising the guanine nucleotide base. The $3^{\prime}$-diphosphate extends away from the core of the protein, towards the solvent and appears to be stabilised only by a long-range 5.5 $\AA$ electrostatic interaction between the lone electron pair on the $\varepsilon$-phosphate of ppGpp and the basic lysine residue K116 (Supplementary Figure S7C). It is worth noting that in the GTP-bound ON state, the switch I/G2 and switch II/G3 flexible loops would aid in stabilising both the catalytic $\mathrm{Mg}^{2+}$ ion and $\gamma$ phosphate $(4,47)$. In our structures there is no electron density corresponding to the $\mathrm{Mg}^{2+}$ and the switch I/G2 loop is unresolved, likely due to innate flexibility when not contacting a $\gamma$-phosphate. Additionally, the switch II/G3 loop does not appear to form hydrogen-bonds or electrostatic interactions with the ligand.

\section{ppGpp-bound RsgA mimics the GDP-bound OFF-state conformation}

For RsgA, a catalytic histidine residue is located within the switch I/G2 loop, two residues upstream of the conserved G2 threonine (4). Therefore, correct docking of this region upon binding to either GTP or the 16S rRNA is thought to be instrumental for GTPase activity. It has also been previously proposed by Pausch et al. (6) that for RbgA, the $3^{\prime}$-diphosphate of (p)ppGpp prevents the movement of switch I/G2 into the ON conformation necessary for GTP hydrolysis and ribosome binding, explaining why the GTPase is incapable of hydrolysing (p)ppGpp in a similar manner to GTP (6). In order to determine whether a similar steric inhibition is occurring for RsgA, we compared our apo and ppGpp-bound structures with available structures of RsgA homologues, namely Aquifex aeolicus YjeQ bound to GDP (PDB: 2YV5) and E. coli YjeQ complexed with both the 30S subunit and GMPPNP (PDB: 5 UZ4 (47)) (Figure 6). Importantly, in both of these available structures, the switch I/G2 loops were partially resolved (Figure 6A, 6B). Despite a similar overall fold of the G-domain, the switch I/G2 loop in the GDP-bound structure appears to extend distally from the main body of the protein, far from the associated ligand (Figure 6A). Contrary to this, the GMPPNP-bound structure features a fully docked Switch I/G2 loop, positioned adjacent to the bound ligand and the binding site of the $\mathrm{Mg}^{2+}$ ion, although the $\mathrm{Mg}^{2+}$ ion itself is not resolved. Crucially, in this conformation, the docked switch I/G2 loop occupies the same space that the $3^{\prime}$-diphosphate moiety of ppGpp would (Figure 6B, 6D). Additionally, the switch II/G3 loop conformation differs between the GDP- and GMPPNP-bound structures, being extended towards the $\mathrm{Y}$-phosphate of GMPPNP in the latter. When compared to our apo (Figure 6C) and ppGpp- 
bound (Figure 6D) structures, the switch II/G3 region appears highly similar to that of the GDP-bound structure, leading us to hypothesise that the switch I/G2 loop will also adopt a similar conformation to the GDP-bound state due to steric inhibition by ppGpp. This lack of docking of switch I/G2 would inhibit GTPase activity by preventing proper docking of the catalytic histidine within switch I (4), coordination of the $\mathrm{Mg}^{2+}$ cofactor by the $\mathrm{G} 2$ threonine (6), and subsequent interaction with the $\mathrm{Y}$-phosphate of GTP.

\section{Displacement of the G2 loop by (p)ppGpp inhibits RA-GTPase-ribosome interactions}

The structure of RsgA in the GMPPNP-bound ON state has only ever been solved when associated with the 30 ribosomal subunit suggesting it is stabilised in this conformation $(4,47)$. In order to assess the role of the switch I/G2 loop in ribosome association, we performed computational $\mathrm{C}_{\alpha}$ alignments of both the available GDP-bound (PDB: 2YV5) and our ppGpp-bound structures with the GMPPNP-bound RsgA-30S ribosome complex (PDB: 5UZ4) (Figure 7A-C). It has previously been shown that each of the 3 domains of RsgA interact with rRNA to provide a stable docking interaction (Figure 5C) (4), and that for E. coli RsgA, the switch I/G2 loop and a $\beta 6, \beta 7$-hairpin clamp around h44, contacting the minor and major groove respectively (Figure 7A). However, when the GDP-bound OFF-state structure from A. aeolicus is superimposed in place of the GMPPNP structure, it appears that the switch I/G2 loop is positioned in such a way that would cause steric clashing between the phosphate backbone of h44 (Figure 7B). Likewise, the expected position of the switch I/G2 loop in the ppGpp-bound model would lead to similar steric clashing, with the 3'-diphosphate moiety of ppGpp preventing the switch I /G2 loop adopting the active conformation (Figure $7 \mathrm{C}$ ). While it is important to stress that this modelling is performed using protein models and $30 \mathrm{~S}$ subunits from separate organisms, this leads us to hypothesise that the misalignment of the switch I/G2 loop and subsequent steric clashing between the RA-GTPase and h44 of the 16S rRNA could be responsible for (p)ppGpp-mediated inhibition of RAGTPase association to the ribosome. We suggest that this region is not directly responsible for promoting subunit docking, however that the switch I region instead forms electrostatic interactions with conformationally mature $\mathrm{h} 44$ and h45 rRNA following ribosome association, enabling positioning of the switch I/G2 loop in a catalytically active conformation when the mature rRNA conformation is reached. These interactions and the subsequent loop rearrangement may represent the slow stabilisation step $\left(k_{2}\right)$ observed in our stopped flow analysis (Figure 3).

\section{DISCUSSION}

The stringent response is a multi-faceted stress coping mechanism, ubiquitously used throughout the Bacteria to cope with nutrient starvation conditions. Recent transcriptomics data has highlighted the diversity and complexity of this response, with 757 genes being differentially regulated within 5 minutes of (p)ppGpp induction (15). For Gram-positive bacteria, the regulation of transcription by (p)ppGpp is intricately linked to purine nucleotide levels, which are impacted in a number of ways (48). Upon 
induction of the stringent response, GTP/GDP and ATP levels decrease as they are utilised by (p)ppGpp synthetase enzymes (12). Furthermore, once produced (p)ppGpp directly inhibits a number of enzymes involved in the guanylate and adenylate synthesis pathways, further reducing GTP/GDP levels $(38,49)$. All of this results in a shift from high GTP/GDP and low (p)ppGpp levels in fast growing cells, to low GTP/GDP and high (p)ppGpp in nutritionally starved cells. For $S$. aureus, the impacts of this are wide-reaching, affecting transcription initiation (39), enzyme activities (50) and, as we show here, the regulation of the activity of RA-GTPases by tuning their capacity to interact with ribosomal subunits.

In the present work, we examine the nucleotide binding preferences of RA-GTPases, and the consequences of this binding on regulating the interactions of RA-GTPases with the ribosome. Cycling between the GTP-bound ON and GDP-bound OFF states is critically important for RA-GTPases, as it enables these proteins to act as molecular checkpoints of ribosome assembly. Here we show that RAGTPases bind to guanosine nucleotides competitively and with differing affinities, with GDP and ppGpp binding with up to 6-times greater affinity than their $5^{\prime}$ trinucleotide-containing counterparts GTP and pppGpp (Supplementary Table S4). The consequence of differing nucleotide-bound states for interactions with ribosomal subunits are significant. We observe that GTP binding is required to promote RA-GTPase/ribosome interactions (Figure $2 \& 3$ ). Indeed, the binding of apo RbgA and $\mathrm{HfIX}$ to the $50 \mathrm{~S}$ subunit was almost undetectable by stopped-flow fluorescence (Figure $3 \mathrm{~B}, 3 \mathrm{C}$ ), although Era and $\mathrm{HflX}$ did demonstrate background binding to the $30 \mathrm{~S}$ and $50 \mathrm{~S}$ subunits respectively by western immunoblotting. A cryo-electron micrograph (cryo-EM) structure of Era binding to the $30 \mathrm{~S}$ subunit has previously been solved (40), demonstrating that this GTPase can bind in the apo form. Upon induction of the stringent response, levels of ( $p$ )ppGpp in the cell rise, swiftly becoming the dominant guanosine nucleotide in the cell $(12,51)$, causing ( $p$ )ppGpp to out-compete GTP for occupancy of the nucleotide binding site (Figure 1C, Supplementary Figure S2D-F), and resulting in reduced association of RAGTPases to their target ribosomal subunit and thus reduced production of mature 705 ribosomes (Figure 2, $3 \& 4$ ). It has been previously shown that, opposite to our observations regarding ribosome assembly factors, ppGpp binding enhances the affinity of the (p)ppGpp-binding RA-GTPase ObgE to the $50 \mathrm{~S}$ subunit (52). This may reflect the proposed role of ObgE as a $50 \mathrm{~S}$ based late-stage antiassociation factor (52) which would benefit from enhanced affinity for the $50 \mathrm{~S}$ in the ppGpp-bound state to prevent subunit joining and $70 S$ formation. Unfortunately, we were unable to purify enough ObgE to compare using our system. Structural studies indicate that ppGpp-bound RsgA and RbgA most likely mimic the GDP-bound OFF state (Figure $5 \& 6)(6)$, with (p)ppGpp inhibiting both GTPase activity and ribosome association by displacement of the switch I/G2 loop into an OFF-state conformation not compatible with stable ribosome subunit interaction (Figure 7). Given the reaction scheme determined by stopped-flow fluorescence, it is possible that the slower stabilisation step $\left(k_{2}\right)$ observed when HflX is in the ppGpp-bound state compared to the GTP-bound state could be due to improper loop docking following association of the RNA-binding domain(s) with the ribosome, leading to dissociation.

Altogether, our data favour a model (Figure 8) whereby in unstressed growing cells, GTP is the predominant nucleotide and induces the RA-GTPase ON-state conformation. Binding of the enzymes to each individual ribosomal subunit follows in order to promote a processing event. Following this, GTP 
is hydrolysed to GDP, inducing formation of the OFF state and subsequent dissociation. Upon cell starvation, the concentration of (p)ppGpp in the cell rises sharply, where it can out-compete GTP for binding to the RA-GTPases. The increase in (p)ppGpp not only inhibits the GTPase activity, but also negatively impacts the stability of RA-GTPase-ribosome interactions, reducing biogenesis and slowing growth. With its high concentration and affinity, ( $p$ )ppGpp could remain bound to RA-GTPases, preventing further ribosome biogenesis during low-energy conditions, yet preserving a pool of enzymes ready for rapid resumption of growth upon restoration of the proliferative state.

All of the above analyses suggest that both pppGpp and ppGpp mimic the GDP-bound OFF conformation, an assertion that we support by solving the crystal structure of RsgA in the apo and ppGpp-bound states (Figure $5 \& 6$ ). These structures are in line with the OFF-state conformations observed by Pausch et al for RbgA in complex with (p)ppGpp (6). Similar to our RsgA-ppGpp structure, the diphosphate moieties of ppGpp bound by RbgA are in the elongated conformation (6), where the 3' and 5 '-phosphate moieties face away from each other. This configuration is not consistent among all (p)ppGpp-binding proteins or even among RA-GTPases. For example, the E. coli RA-GTPases BipA and ObgE bind to ppGpp in a ring-like conformation (53-55), in which the $3^{\prime}$ and $5^{\prime}$ phosphate moieties point towards each other. While no structural reasoning for this difference in conformation is known, aside from to extend the breadth of responses controlled by (p)ppGpp, it has been suggested that proteins which bind (p)ppGpp in the ring-like conformation have 10-fold lower inhibitory constants and dissociation constants than those which bind in the elongated conformation $(56,57)$. This could potentially influence the temporal or energetic threshold during the stringent response where a certain protein becomes inhibited, based on decreasing concentrations of GTP and increasing concentrations of (p)ppGpp (38).

Ribosomal rRNA production and biogenesis are not the only aspects of protein synthesis that (p)ppGpp regulates, given its ability to bind to the bacterial initiation factor 2 (IF2), elongation factor Tu (EF-Tu), elongation factor $\mathrm{G}$ (EFG), elongation factor Ts (EFTs) and release factor 3 (RF3) (58-62). In each case, competitive binding of (p)ppGpp to these GTPases results in an inhibition of activity and reduction of the elongation cycle. Unlike RA-GTPases involved in subunit maturation, both IF2 and EFG bind to GTP, GDP and (p)ppGpp with similar affinity $(59,60,63)$, albeit with EFG demonstrating an overall lower affinity. Furthermore, IF2 binding to (p)ppGpp within the $30 \mathrm{~S}$ pre-initiation complex alters the mRNA binding preference, enabling permissive translation of certain mRNAs such as tufA encoding EF-Tu (58), which may fine-tune the proteome on a translational level to better enable survival of nutrient deprivation.

With this work we have used complementary techniques to demonstrate that (p)ppGpp prevents stable association of RA-GTPases to the ribosome, both in vitro and within the bacterial cell. This is achieved by these enzymes having a stronger affinity for ppGpp over GTP, with ppGpp interactions holding these enzymes in an OFF-state conformation. Consequently, this imparts delays to $70 \mathrm{~S}$ ribosome assembly, which in turn contributes to the growth defects that are observed upon induction of the stringent response. Altogether, we highlight RA-GTPases-(p)ppGpp interactions as important regulators of stringent response-mediated growth control. 
bioRxiv preprint doi: https://doi.org/10.1101/2021.01.19.427108; this version posted January 19, 2021. The copyright holder for this preprint (which was not certified by peer review) is the author/funder, who has granted bioRxiv a license to display the preprint in perpetuity. It is made available under aCC-BY 4.0 International license.

\section{DATA AVAILABILITY}

The coordinates and electron density maps of RsgA-apo and RsgA-ppGpp have been deposited in the Protein Data Bank in Europe (PDBe) (https://www.ebi.ac.uk/pdbe/node/1) under accession codes 6ZJO and $6 Z \mathrm{HL}$ respectively.

\section{SUPPLEMENTARY DATA}

Supplementary data are available at NAR Online.

\section{FUNDING}

This work was supported by a Sir Henry Dale Fellowship jointly funded by the Wellcome Trust and the Royal Society [https://wellcome.ac.uk:104110/Z/14/Z to RMC]; a Lister Institute Research Prize 2018 [to RMC]; the MRC Discovery Medicine North Doctoral Training Partnership [MR/N013840/1 to DJB]; a BBSRC award [BBSRC BB/T008032/1 to TDC]; the Fondo Nacional de Desarrollo Científico, Tecnológico y de Innovación Tecnológica grant [154-2017-Fondecyt to PM]; and by equipment from the InnóvatePerú grant [297-INNOVATEPERU-EC-2016 to PM].

\section{CONFLICTS OF INTEREST}

The authors declare no conflicts of interest. 


\section{REFERENCES}

1. $\quad$ Davis, J.H., Tan, Y.Z., Carragher, B., Potter, C.S., Lyumkis, D. and Williamson, J.R. (2016) Modular Assembly of the Bacterial Large Ribosomal Subunit. Cell, 167, 1610-1622.

2. Britton, R.A. (2009) Role of GTPases in Bacterial Ribosome Assembly. Annual Review of Microbiology, 63, 155-176.

3. Tu, C., Zhou, X., Tropea, J.E., Austin, B.P., Waugh, D.S., Court, D.L. and Ji, X. (2009) Structure of Era in complex with the $3^{\prime}$ end of $16 \mathrm{~S}$ rRNA: Implications for ribosome biogenesis. Proceedings of the National Academy of Sciences, 106, 14843-14848.

4. López-Alonso, J.P., Kaminishi, T., Kikuchi, T., Hirata, Y., Iturrioz, I., Dhimole, N., Schedlbauer, A., Hase, Y., Goto, S., Kurita, D. et al. (2017) RsgA couples the maturation state of the $30 \mathrm{~S}$ ribosomal decoding center to activation of its GTPase pocket. Nucleic Acids Res, 45, 69456959.

5. Dey, S., Biswas, C. and Sengupta, J. (2018) The universally conserved GTPase HfIX is an RNA helicase that restores heat-damaged Escherichia coli ribosomes. Journal of Cell Biology, 217, 2519-2529.

6. Pausch, P., Steinchen, W., Wieland, M., Klaus, T., Freibert, S.A., Altegoer, F., Wilson, D.N. and Bange, G. (2018) Structural basis for (p)ppGpp-mediated inhibition of the GTPase RbgA. Journal of Biological Chemistry, 293, 19699-19709.

7. Verstraeten, N., Fauvart, M., Versees, W. and Michiels, J. (2011) The Universally Conserved Prokaryotic GTPases. Microbiology and Molecular Biology Reviews, 75, 507-542.

8. Karbstein, K. (2007) Role of GTPases in ribosome assembly. Biopolymers, 87, 1-11.

9. Vercruysse, M., Köhrer, C., Shen, Y., Proulx, S., Ghosal, A., Davies, B.W., RajBhandary, U.L. and Walker, G.C. (2016) Identification of YbeY-protein interactions involved in 16S rRNA maturation and stress regulation in Escherichia coli. mBio, 7, e01785-01716.

10. Wood, A., Irving, S.E., Bennison, D.J. and Corrigan, R.M. (2019) The (p)ppGpp-binding GTPase Era promotes rRNA processing and cold adaptation in Staphylococcus aureus. PLoS Genetics, 15, e1008346.

11. Cashel, M. (1969) The control of ribonucleic acid synthesis in Escherichia coli. IV. Relevance of unusual phosphorylated compounds from amino acid-starved stringent strains. J Biol Chem, 244, 3133-3141.

12. Varik, V., Oliveira, S.R.A., Hauryliuk, V. and Tenson, T. (2017) HPLC-based quantification of bacterial housekeeping nucleotides and alarmone messengers ppGpp and pppGpp. Sci Rep, 7, 11022.

13. Fiil, N.P., von Meyenburg, K. and Friesen, J.D. (1972) Accumulation and turnover of guanosine tetraphosphate in Escherichia coli. J Mol Biol, 71, 769-783.

14. Gralla, J.D. (2005) Escherichia coli ribosomal RNA transcription: Regulatory roles for ppGpp, NTPs, architectural proteins and a polymerase-binding protein. Molecular Microbiology, 55, 973-977.

15. Sanchez-Vazquez, P., Dewey, C.N., Kitten, N., Ross, W. and Gourse, R.L. (2019) Genomewide effects on Escherichia coli transcription from ppGpp binding to its two sites on RNA polymerase. Proc Natl Acad Sci USA, 116, 8310-8319.

16. Dalebroux, Z.D., Svensson, S.L., Gaynor, E.C. and Swanson, M.S. (2010) ppGpp conjures bacterial virulence. Microbiol Mol Biol Rev, 74, 171-199.

17. Corrigan, R.M., Bellows, L.E., Wood, A. and Gründling, A. (2016) ppGpp negatively impacts ribosome assembly affecting growth and antimicrobial tolerance in Gram-positive bacteria. Proceedings of the National Academy of Sciences, 113, E1710-E1719.

18. Levdikov, V.M., Blagova, E.V., Brannigan, J.A., Cladière, L., Antson, A.A., Isupov, M.N., Séror, S.J. and Wilkinson, A.J. (2004) The crystal structure of YloQ, a circularly permuted GTPase essential for Bacillus subtilis viability. Journal of Molecular Biology, 340, 767-782.

19. Guo, Q., Yuan, Y., Xu, Y., Feng, B., Liu, L., Chen, K., Sun, M., Yang, Z., Lei, J. and Gao, N. (2011) Structural basis for the function of a small GTPase RsgA on the 30S ribosomal subunit maturation revealed by cryoelectron microscopy. Proceedings of the National Academy of Sciences, 108, 13100-13105.

20. Firpo, M.A., Connelly, M.B., Goss, D.J. and Dahlberg, A.E. (1996) Mutations at two invariant nucleotides in the 3'-minor domain of Escherichia coli $16 \mathrm{~S}$ rRNA affecting translational initiation and initiation factor 3 function. $J$ Biol Chem, 271, 4693-4698.

21. Do, J.K., Jun, Y.J., Yoon, H.J. and Se, W.S. (2008) Crystal structure of YlqF, a circularly permuted GTPase: Implications for its GTPase activation in 50S ribosomal subunit assembly. Proteins: Structure, Function and Genetics, 72, 1363-1370. 
22. Fischer, J.J., Coatham, M.L., Bear, S.E., Brandon, H.E., De Laurentiis, E.I., Shields, M.J. and Wieden, H.J. (2012) The ribosome modulates the structural dynamics of the conserved GTPase HfIX and triggers tight nucleotide binding. Biochimie, 94, 1647-1659.

23. Basu, A. and Yap, M.N. (2017) Disassembly of the Staphylococcus aureus hibernating $100 \mathrm{~S}$ ribosome by an evolutionarily conserved GTPase. Proc Natl Acad Sci U S A, 114, E8165e8173.

24. Achila, D., Gulati, M., Jain, N. and Britton, R.A. (2012) Biochemical characterization of ribosome assembly gtpase RbgA in Bacillus subtilis. Journal of Biological Chemistry, 287, 8417-8423.

25. Bremer, H. and Dennis, P.P. (2008) Modulation of Chemical Composition and Other Parameters of the Cell at Different Exponential Growth Rates. EcoSal Plus, 3.

26. Daigle, D.M.a.B.E.D. (2004) Studies of the Interaction of Itextless i Itextgreater Escherichia coli Itextless /i Itextgreater YjeQ with the Ribosome In Vitro. Journal of Bacteriology, 186, 1381-1387.

27. Loh, P.C., Morimoto, T., Matsuo, Y., Oshima, T. and Ogasawara, N. (2007) The GTP-binding protein YqeH participates in biogenesis of the $30 \mathrm{~S}$ ribosome subunit in Bacillus subtilis. Genes Genet Syst, 82, 281-289.

28. Emsley, P. and Cowtan, K. (2004) Coot: model-building tools for molecular graphics. Acta Crystallogr D Biol Crystallogr, 60, 2126-2132.

29. Emsley, P., Lohkamp, B., Scott, W.G. and Cowtan, K. (2010) Features and development of Coot. Acta Crystallogr D Biol Crystallogr, 66, 486-501.

30. Winter, G. (2010) xia2: an expert system for macromolecular crystallography data reduction. Journal of Applied Crystallography, 43, 186-190.

31. McCoy, A.J., Grosse-Kunstleve, R.W., Adams, P.D., Winn, M.D., Storoni, L.C. and Read, R.J. (2007) Phaser crystallographic software. J Appl Crystallogr, 40, 658-674.

32. Winn, M.D., Ballard, C.C., Cowtan, K.D., Dodson, E.J., Emsley, P., Evans, P.R., Keegan, R.M., Krissinel, E.B., Leslie, A.G., McCoy, A. et al. (2011) Overview of the CCP4 suite and current developments. Acta Crystallogr D Biol Crystallogr, 67, 235-242.

33. Murshudov, G.N., Vagin, A.A. and Dodson, E.J. (1997) Refinement of macromolecular structures by the maximum-likelihood method. Acta Crystallogr D Biol Crystallogr, 53, 240-255.

34. Williams, C.J., Headd, J.J., Moriarty, N.W., Prisant, M.G., Videau, L.L., Deis, L.N., Verma, V., Keedy, D.A., Hintze, B.J., Chen, V.B. et al. (2018) MolProbity: More and better reference data for improved all-atom structure validation. Protein Sci, 27, 293-315.

35. Milon, P., Konevega, A.L., Peske, F., Fabbretti, A., Gualerzi, C.O. and Rodnina, M.V. (2007) Transient kinetics, fluorescence, and FRET in studies of initiation of translation in bacteria. Methods Enzymol, 430, 1-30.

36. Roelofs, K.G., Wang, J., Sintim, H.O. and Lee, V.T. (2011) Differential radial capillary action of ligand assay for high-throughput detection of protein-metabolite interactions. Proc Natl Acad Sci U S A, 108, 15528-15533.

37. Rensland, H., John, J., Linke, R., Simon, I., Schlichting, I., Wittinghofer, A. and Goody, R.S. (1995) Substrate and product structural requirements for binding of nucleotides to H-ras p21: the mechanism of discrimination between guanosine and adenosine nucleotides. Biochemistry, 34, 593-599.

38. Kriel, A., Bittner, A.N., Kim, S.H., Liu, K., Tehranchi, A.K., Zou, W.Y., Rendon, S., Chen, R., Tu, B.P. and Wang, J.D. (2012) Direct regulation of GTP homeostasis by (p)ppGpp: a critical component of viability and stress resistance. Mol Cell, 48, 231-241.

39. Kästle, B., Geiger, T., Gratani, F.L., Reisinger, R., Goerke, C., Borisova, M., Mayer, C. and Wolz, C. (2015) rRNA regulation during growth and under stringent conditions in Staphylococcus aureus. Environmental Microbiology, 17, 4394-4405.

40. Sharma, M.R., Barat, C., Wilson, D.N., Booth, T.M., Kawazoe, M., Hori-Takemoto, C., Shirouzu, M., Yokoyama, S., Fucini, P. and Agrawal, R.K. (2005) Interaction of Era with the $30 S$ ribosomal subunit implications for $30 \mathrm{~S}$ subunit assembly. Mol Cell, 18, 319-329.

41. Waterhouse, A., Bertoni, M., Bienert, S., Studer, G., Tauriello, G., Gumienny, R., Heer, F.T., de Beer, T.A.P., Rempfer, C., Bordoli, L. et al. (2018) SWISS-MODEL: homology modelling of protein structures and complexes. Nucleic Acids Res, 46, W296-W303.

42. Inoue, K., Alsina, J., Chen, J. and Inouye, M. (2003) Suppression of defective ribosome assembly in a rbfA deletion mutant by overexpression of Era, an essential GTPase in Escherichia coli. Mol Microbiol, 48, 1005-1016.

43. Reiss, S., Pane-Farre, J., Fuchs, S., Francois, P., Liebeke, M., Schrenzel, J., Lindequist, U., Lalk, M., Wolz, C., Hecker, M. et al. (2012) Global analysis of the Staphylococcus aureus response to mupirocin. Antimicrob Agents Chemother, 56, 787-804. 
44. Vetter, I.R. and Wittinghofer, A. (2001) The guanine nucleotide-binding switch in three dimensions. Science, 294, 1299-1304.

45. Theobald, D.L., Mitton-Fry, R.M. and Wuttke, D.S. (2003) Nucleic acid recognition by OB-fold proteins. Annu Rev Biophys Biomol Struct, 32, 115-133.

46. Font, J. and Mackay, J.P. (2010) Beyond DNA: zinc finger domains as RNA-binding modules. Methods Mol Biol, 649, 479-491.

47. Razi, A., Guarné, A. and Ortega, J. (2017) The cryo-EM structure of YjeQ bound to the $30 \mathrm{~S}$ subunit suggests a fidelity checkpoint function for this protein in ribosome assembly. Proc Natl Acad Sci U S A, 114, E3396-E3403.

48. Krasny, L. and Gourse, R.L. (2004) An alternative strategy for bacterial ribosome synthesis: Bacillus subtilis rRNA transcription regulation. Embo J, 23, 4473-4483.

49. Wang, B., Dai, P., Ding, D., Del Rosario, A., Grant, R.A., Pentelute, B.L. and Laub, M.T. (2018) Affinity-based capture and identification of protein effectors of the growth regulator ppGpp. Nat Chem Biol, 15, 141-150.

50. Rymer, R.U., Solorio, F.A., Tehranchi, A.K., Chu, C., Corn, J.E., Keck, J.L., Wang, J.D. and Berger, J.M. (2012) Binding mechanism of metalNTP substrates and stringent-response alarmones to bacterial DnaG-type primases. Structure, 20, 1478-1489.

51. Zborníková, E., Knejzlík, Z., Hauryliuk, V., Krásný, L. and Rejman, D. (2019) Analysis of nucleotide pools in bacteria using HPLC-MS in HILIC mode. Talanta, 205, 120161.

52. Feng, B., Mandava, C.S., Guo, Q., Wang, J., Cao, W., Li, N., Zhang, Y., Wang, Z., Wu, J., Sanyal, S. et al. (2014) Structural and functional insights into the mode of action of a universally conserved Obg GTPase. PLoS Biol, 12, e1001866.

53. Buglino, J., Shen, V., Hakimian, P. and Lima, C.D. (2002) Structural and biochemical analysis of the Obg GTP binding protein. Structure, 10, 1581-1592.

54. Fan, H., Hahm, J., Diggs, S., Perry, J.J. and Blaha, G. (2015) Structural and Functional Analysis of BipA, a Regulator of Virulence in Enteropathogenic Escherichia coli. J Biol Chem, 290, 20856-20864.

55. Kumar, V., Chen, Y., Ero, R., Ahmed, T., Tan, J., Li, Z., Wong, A.S., Bhushan, S. and Gao, Y.G. (2015) Structure of BipA in GTP form bound to the ratcheted ribosome. Proc Natl Acad Sci U S A, 112, 10944-10949.

56. Steinchen, W.a.B.G. (2016) The magic dance of the alarmones (p)ppGpp. Molecular Microbiology, 101, 531--544.

57. Cuthbert, B.J., Ross, W., Rohlfing, A.E., Dove, S.L., Gourse, R.L., Brennan, R.G. and Schumacher, M.A. (2017) Dissection of the molecular circuitry controlling virulence in. Genes Dev, 31, 1549-1560.

58. Vinogradova, D.S., Zegarra, V., Maksimova, E., Nakamoto, J.A., Kasatsky, P., Paleskava, A., Konevega, A.L. and Milon, P. (2020) How the initiating ribosome copes with ppGpp to translate mRNAs. PLoS Biol, 18, e3000593.

59. Milon, P., Tischenko, E., Tomsic, J., Caserta, E., Folkers, G., La Teana, A., Rodnina, M.V., Pon, C.L., Boelens, R. and Gualerzi, C.O. (2006) The nucleotide-binding site of bacterial translation initiation factor 2 (IF2) as a metabolic sensor. Proc Natl Acad Sci U S A, 103, 1396213967.

60. Mitkevich, V.A., Ermakov, A., Kulikova, A.A., Tankov, S., Shyp, V., Soosaar, A., Tenson, T., Makarov, A.A., Ehrenberg, M. and Hauryliuk, V. (2010) Thermodynamic characterization of ppGpp binding to EF-G or IF2 and of initiator tRNA binding to free IF2 in the presence of GDP, GTP, or ppGpp. J Mol Biol, 402, 838-846.

61. Kihira, K., Shimizu, Y., Shomura, Y., Shibata, N., Kitamura, M., Nakagawa, A., Ueda, T., Ochi, K. and Higuchi, Y. (2012) Crystal structure analysis of the translation factor RF3 (release factor 3). FEBS Lett, 586, 3705-3709.

62. Rojas, A.M., Ehrenberg, M., Andersson, S.G. and Kurland, C.G. (1984) ppGpp inhibition of elongation factors Tu, G and Ts during polypeptide synthesis. Mol Gen Genet, 197, 36-45.

63. Hauryliuk, V., Mitkevich, V.A., Eliseeva, N.A., Petrushanko, I.Y., Ehrenberg, M. and Makarov, A.A. (2008) The pretranslocation ribosome is targeted by GTP-bound EF-G in partially activated form. Proc Natl Acad Sci U S A, 105, 15678-15683.

64. Traut, T.W. (1994) Physiological concentrations of purines and pyrimidines. Mol Cell Biochem, 140, 1-22. 


\section{FIGURES}

A

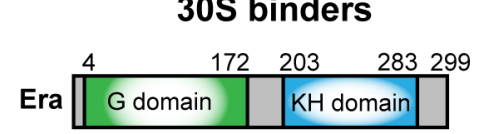

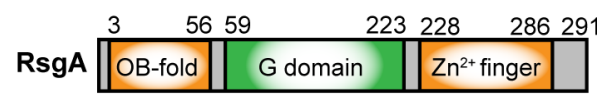

B

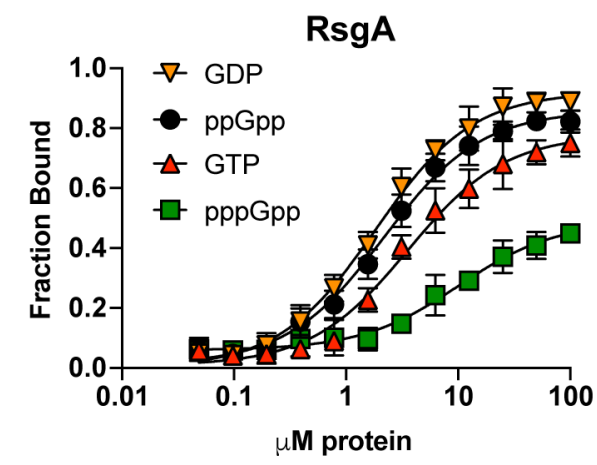

50 binders
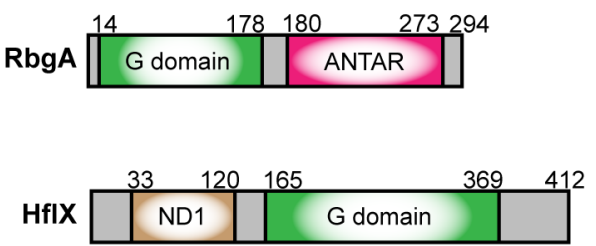

C

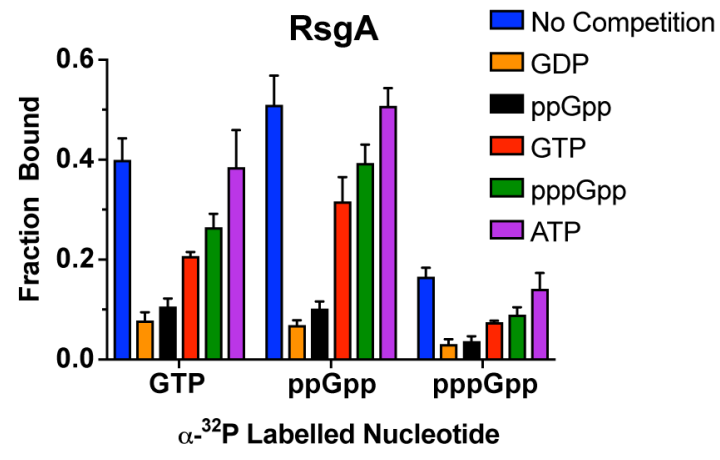

Figure 1. Nucleotide binding characteristics of RA-GTPases. A) Schematic representation of the domain structure of Era, RsgA, RbgA and HflX from S. aureus. The conserved GTPase domain (G domain) is coloured in green and accessory domains are shown. B) Determination of binding affinities and $K_{d}$ values for ${ }^{32} \mathrm{P}$-labelled GTP, GDP, ppGpp and pppGpp with purified recombinant RsgA using DRaCALA as previously described (36). Each point is the mean average of at least 3 technical replicates and error bars indicate standard deviation. C) DRaCALA binding assay of recombinant RsgA binding to ${ }^{32}$ P-labelled GTP, ppGpp and pppGpp in the presence of an excess of cold competitor (GTP, GDP, ppGpp, pppGpp or ATP). All experiments were carried out in triplicate, with error bars representing standard deviation. 
A

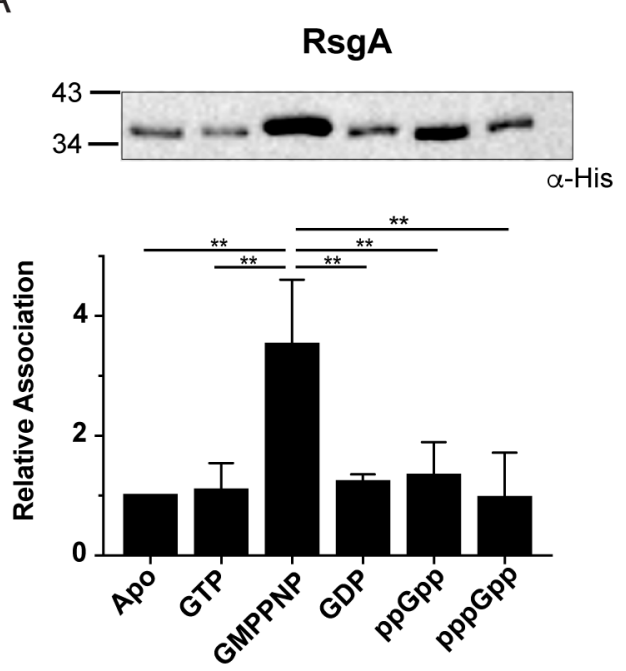

C

Era
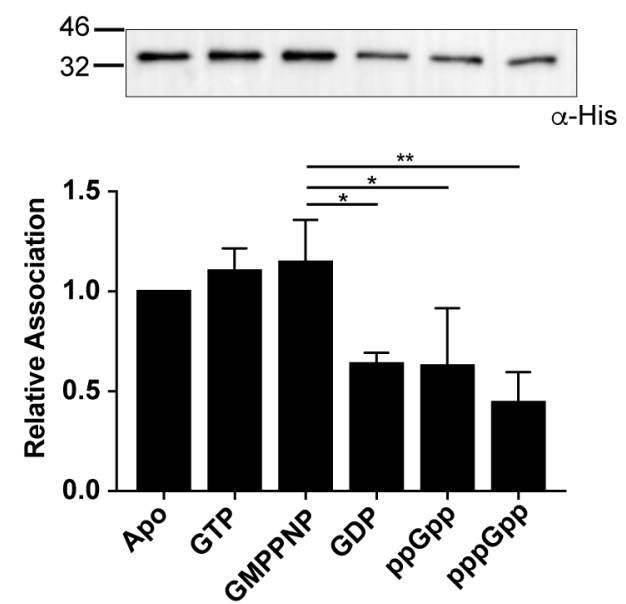

B

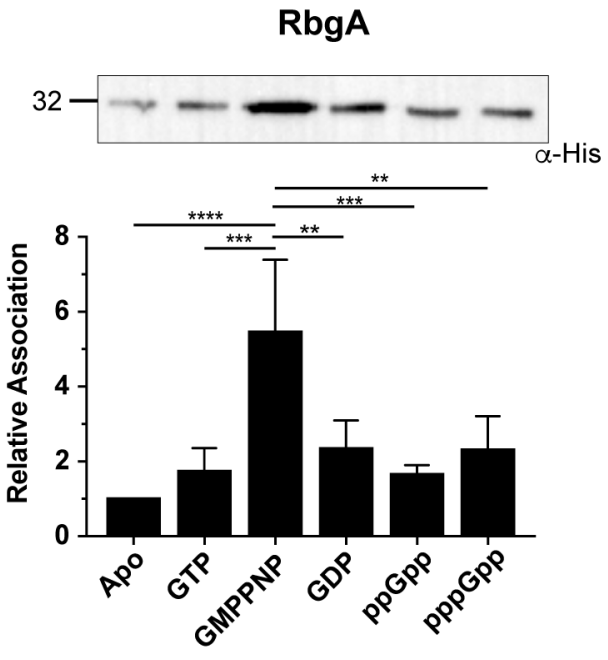

D
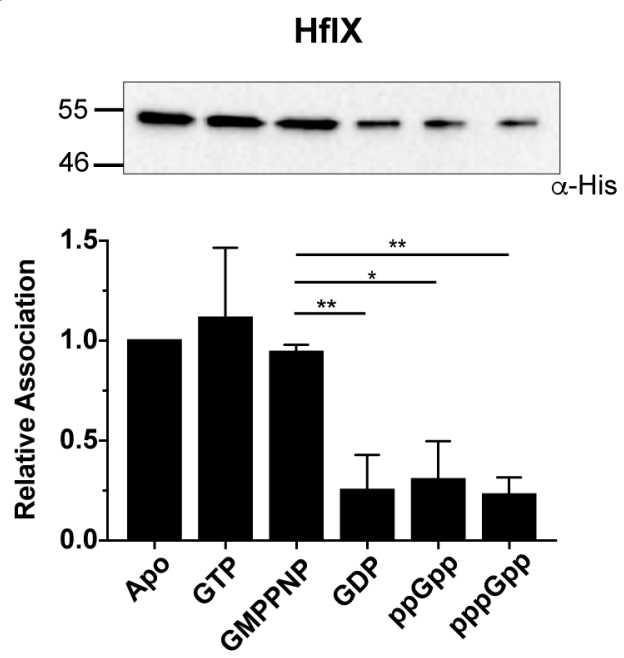

Figure 2. (p)ppGpp and GDP binding reduces RA-GTPase association to the ribosome. A - D) Top: purified $70 \mathrm{~S}$ ribosomes were preincubated with His-tagged A) RsgA, B) RbgA, C) Era and D) HfIX in the absence or presence of GTP, GMPPNP, GDP, ppGpp or pppGpp. Following subunit separation and precipitation, bound proteins were detected using HRP-conjugated $\alpha$-His antibodies. Experiments were carried out in triplicate or quadruplicate and one representative image of each are shown. Bottom: the signal intensities relative to the apo-state of all repeats are plotted with error bars representing standard deviation. Statistical analysis was performed using a one-way ANOVA followed by Tukey's multiple comparison test. ( $P<0.05$, ${ }^{* *} P<0.01$, ${ }^{* * *} P<0.001$, $\left.{ }^{* * *} P<0.0001\right)$. 
A

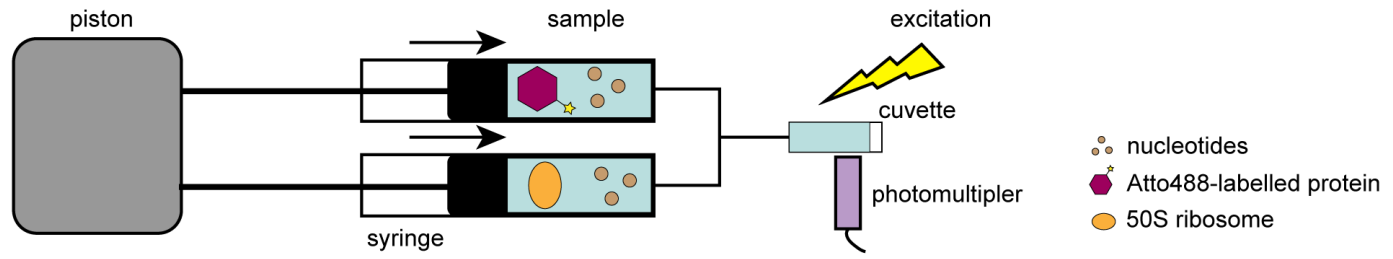

B

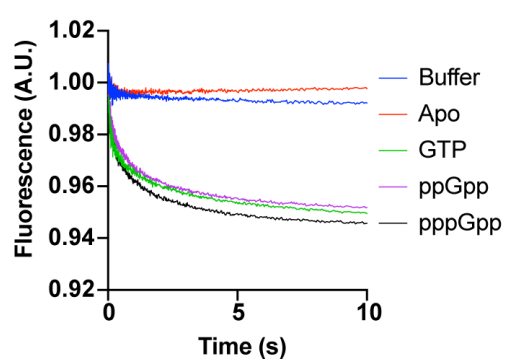

$\mathrm{E}$

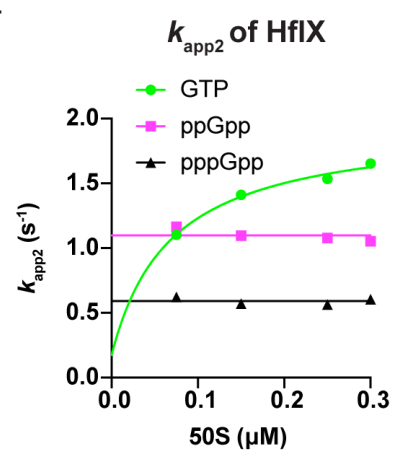

C

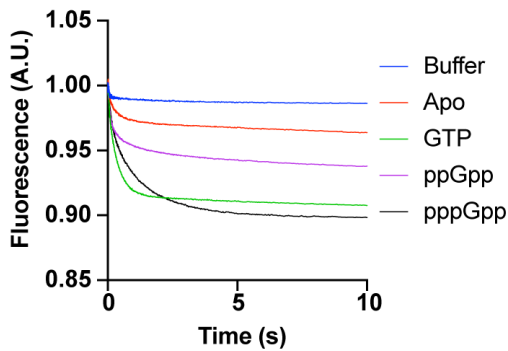

$\mathrm{F}$

Sum of $\mathrm{HflX} \boldsymbol{k}_{\mathrm{app} 1}$ and $\boldsymbol{k}_{\text {app2 }}$

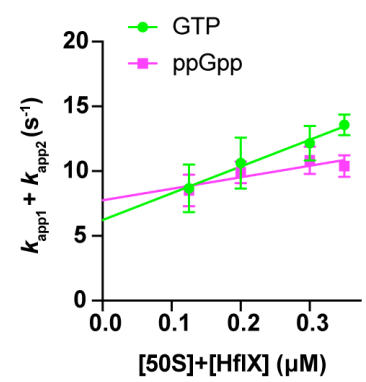

D

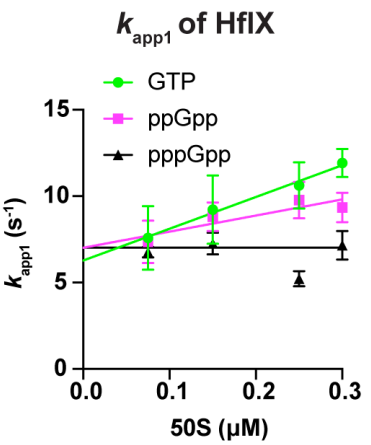

G

Product of $\mathrm{HFIX} \boldsymbol{k}_{\mathrm{app} 1}$ and $\boldsymbol{k}_{\mathrm{app} 2}$

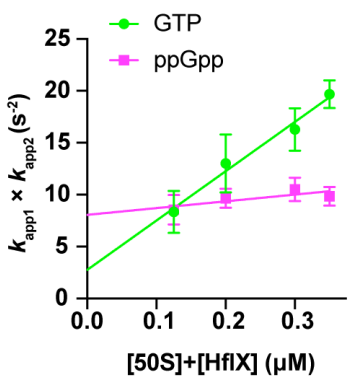

Figure 3. Stopped-flow kinetic parameters of RA-GTPase association to the ribosomal subunits. A) Schematic representation of the experimental setup for stopped-flow analysis. Nucleotides (brown circles), $50 \mathrm{~S}$ subunits (orange oval) and Atto488-labelled RA-GTPases (purple hexagon) are indicated. Arrows indicate the direction of syringe movement. Atto-488 was excited using a $470 \mathrm{~nm}$ LED and fluorescence was detected through a $515 \mathrm{~nm}$ long-pass filter. $B, C$ ) Fluorescent change upon mixing $0.2 \mu \mathrm{M}$ RbgA-Atto488 (B) or HflX-Atto488 (C) with $0.2 \mu \mathrm{M} 50$ S ribosomal subunits in the presence of $100 \mu \mathrm{M}$ GTP, ppGpp and pppGpp or in the Apo state using the stopped flow fluorescence apparatus. Fluorescently labelled protein was also mixed with buffer lacking $50 S$ subunits as a mixing control. Fluorescence of the reaction was tracked using exponential sampling for 10 seconds and each curve is the mean average of at least 5 technical replicates. D) kapp1 dependence on $50 S$ concentration for HflX complexed with GTP (green), ppGpp (pink), pppGpp (black). E) as (D) for the $k_{\text {app2 }}$ dependence. $F$ ) and $G$ ) Sum and product analyses of apparent rates during $\mathrm{HflX}$ association to the $50 \mathrm{~S}$ subunit. 0.05 $\mu \mathrm{M} \mathrm{HfIX-Atto488}$ was mixed with increasing titrations of $50 \mathrm{~S}$ ribosomal subunits over the fluorescently labelled protein, in the presence of $20 \mu \mathrm{M}$ GTP or ppGpp. The resultant traces (Supplementary Figure S4) were analysed by nonlinear regression using two exponential terms. The sum (F) and product (G) of apparent rates $\left(k_{\text {app } 1}(D), k_{\text {app2 }}(E)\right)$ were plotted as a function of the total concentration of the $50 S$ subunits and HflX protein to determine the microscopic constants $k_{1}, k_{-1}, k_{2}$, and $k_{-2}$ (Supplementary 
bioRxiv preprint doi: https://doi.org/10.1101/2021.01.19.427108; this version posted January 19, 2021. The copyright holder for this preprint (which was not certified by peer review) is the author/funder, who has granted bioRxiv a license to display the preprint in perpetuity. It is made available under aCC-BY 4.0 International license.

Table S5) and the resulting dissociation constant $\left(K_{\mathrm{d}}\right)$ (see Methods section). Error bars represent the standard deviation of the apparent rates of 4 or more individual traces $(D, E)$ or the standard error of the two-step analysis $(F, G)$. 
A

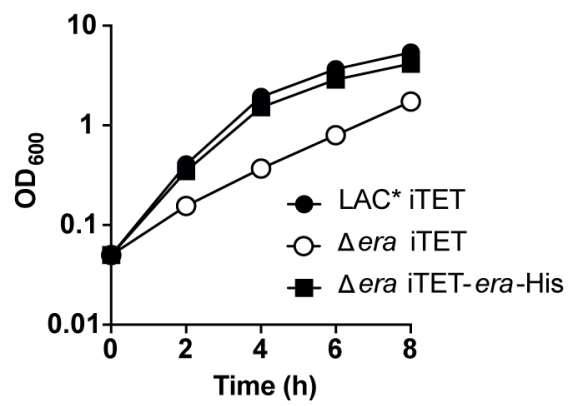

C

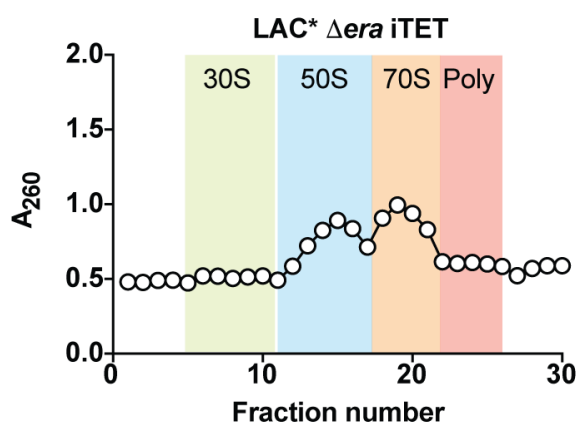

B

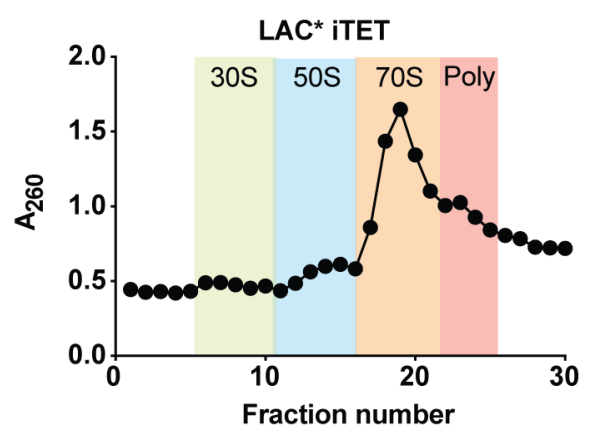

$\mathrm{D}$

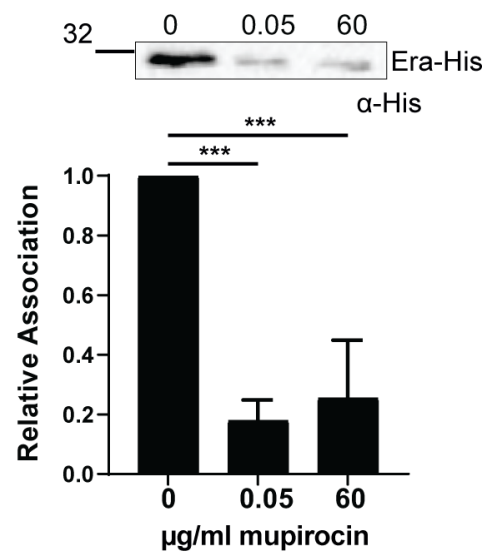

Figure 4. Association of Era to the $30 \mathrm{~S}$ subunit is reduced under stringent conditions. A) Growth curve of $S$. aureus strains LAC* iTET, LAC* $\triangle$ era iTET and LAC* $\triangle$ era iTET-era-His. Overnight cultures were diluted to an $\mathrm{OD}_{600}$ of 0.05 and grown for $8 \mathrm{~h}$ in the presence of $100 \mathrm{ng} / \mu \mathrm{l}$ Atet. Experiments were carried out in triplicate, with error bars representing standard deviation. B, C) Ribosome profiles of the S. aureus strains B) LAC* iTET and C) LAC* $\triangle$ era iTET. RNA content was analysed at an absorbance of $260 \mathrm{~nm}$. All experiments were performed in triplicate, with one representative profile included for each strain. Expected regions for $30 \mathrm{~S}$ subunits (green), $50 \mathrm{~S}$ subunits (blue), $70 \mathrm{~S}$ ribosomes (orange) and polysomes (pink) are highlighted. D) Ribosome association of Era-His from strain LAC* $\triangle$ era iTETera-His. Top: western immunoblot showing the association of Era-His to $30 \mathrm{~S}$ ribosomes. LAC* $\triangle$ era iTET-era-His was grown to an $\mathrm{OD}_{600}$ of 0.5 in the presence of Atet. Cells were either left uninduced or grown in the presence of 0.05 or $60 \mu \mathrm{g} / \mathrm{ml}$ mupirocin for 30 mins to induce the stringent response. Ribosomal subunits were separated and the amount of Era-His associated was detected using HRPconjugated $\alpha$-His antibodies. Experiments were carried out in triplicate and one representative image is shown. Bottom: the mean signal intensities relative to the zero mupirocin sample of all repeats were plotted with error bars representing standard deviation. Statistical analysis was carried out using a oneway ANOVA followed by Tukey's multiple comparisons test $\left({ }^{* * *} P<0.001\right)$. 
A

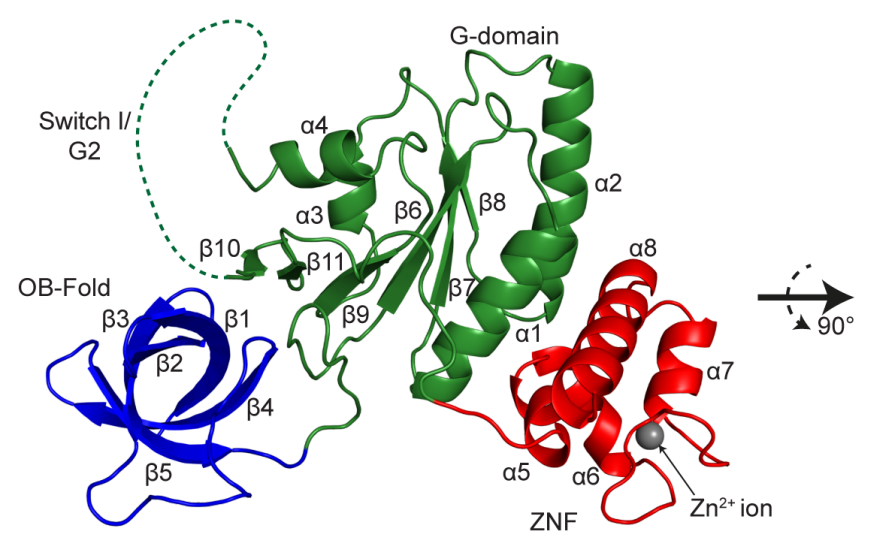

B

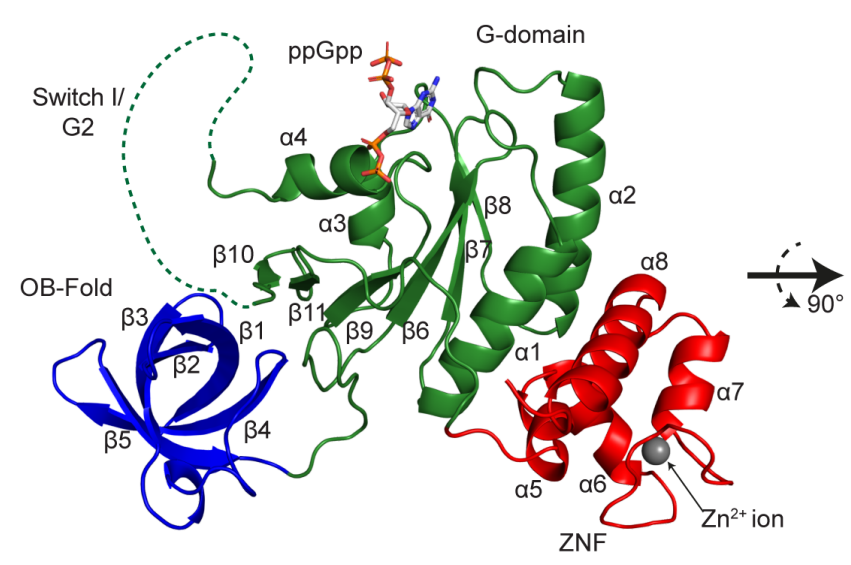

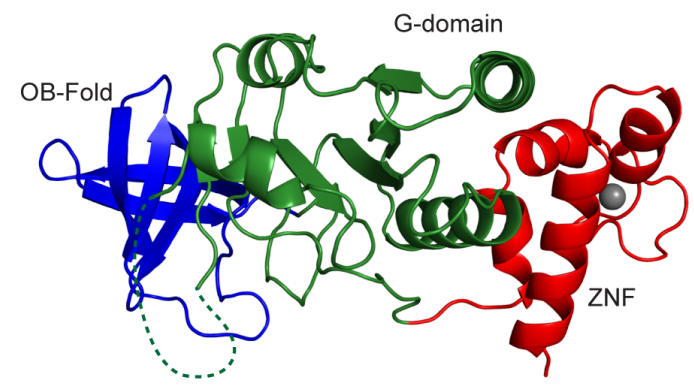

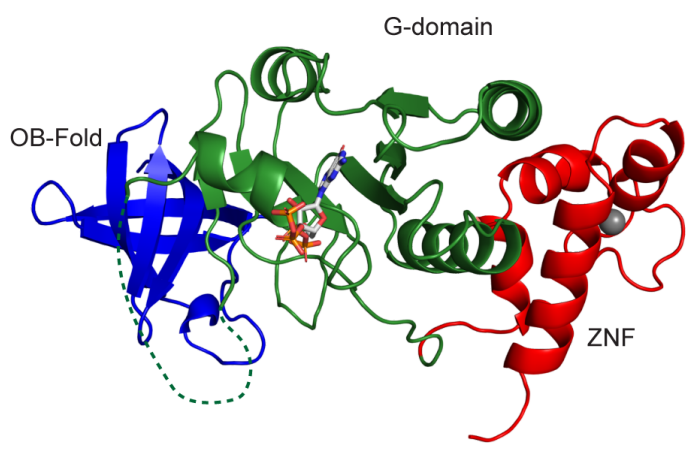

C

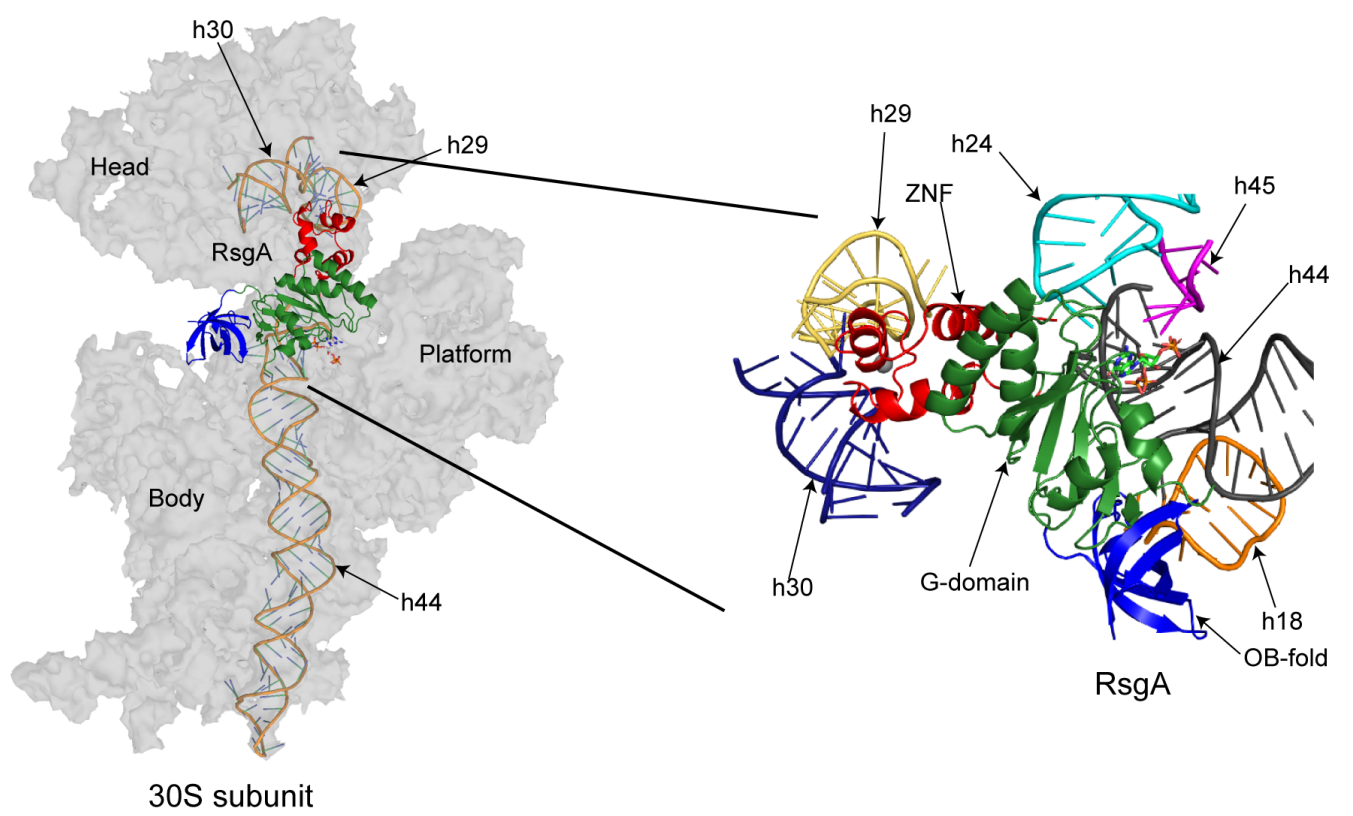

Figure 5. Structure of RsgA in the apo- and ppGpp-bound states. A, B) The crystal structures of RsgA in the apo state (A: PDB: 6ZJO) and bound to ppGpp (B: PDB: 6ZHL). The structures are coloured by 
bioRxiv preprint doi: https://doi.org/10.1101/2021.01.19.427108; this version posted January 19, 2021. The copyright holder for this preprint (which was not certified by peer review) is the author/funder, who has granted bioRxiv a license to display the preprint in perpetuity. It is made available under aCC-BY 4.0 International license.

domain, with the N-terminal OB-fold coloured blue, the central G-domain coloured green and the Cterminal $\mathrm{Zn}^{2+}$-finger $(\mathrm{ZNF})$ domain coloured red. Structural details including $\alpha$-helices, $\beta$-sheets, ligands and domains are labelled. The expected position of the switch I/G2 loops as determined by comparison with RsgA homologues in the GMPPNP-bound state are indicated using a dotted line, despite the lack of electron density surrounding this feature. For both $A$ ) and $B$ ), a $90^{\circ}$ rotation around a horizontal axis is shown. C) The RsgA binding site on the 30S ribosomal subunit. RsgA-ppGpp (PDB: 6ZHL, this study) was overlaid onto the model of YjeQ-GMPPNP (PDB: 5UZ4, chain Z (47)) using $\mathrm{C}_{\alpha}$ alignment, relative to the 30S ribosomal subunit (PDB: $5 \mathrm{UZ4}$, chain A (47)). The RsgA model is shown as a cartoon representation, coloured by domain as above. The 30 S subunit is shown in grey, with interacting rRNA helices shown as cartoon representations to highlight the RsgA recognition sites, as labelled. The bound ppGpp ligand is coloured by atom: carbon, grey; nitrogen, blue; oxygen, red; phosphorous, orange. Inset: a cartoon representation of the rRNA helices which constitute the RsgA binding site on the $30 \mathrm{~S}$ subunit. Target rRNA helices are coloured as follows: $h 24$, cyan; $h 18$, orange; $h 29$, yellow; h30, navy blue; h44, grey; h45, magenta. 
A

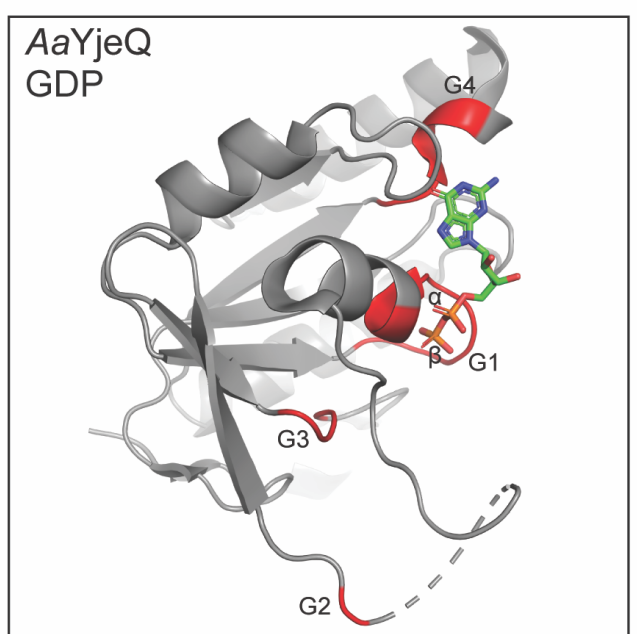

C

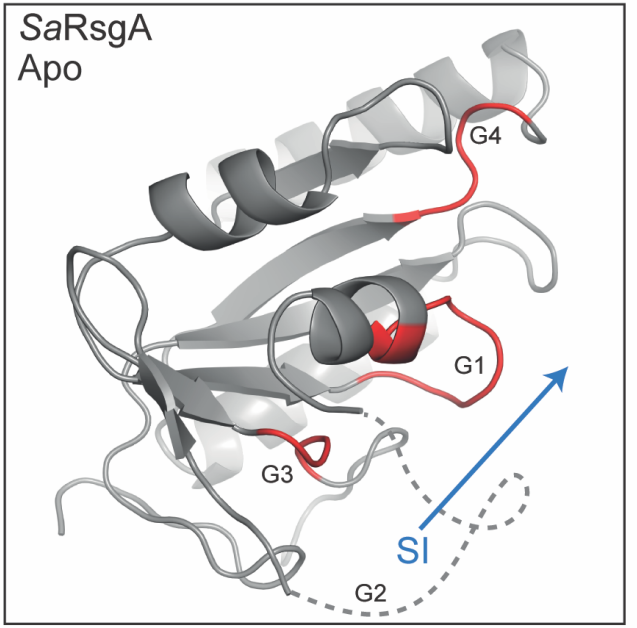

B

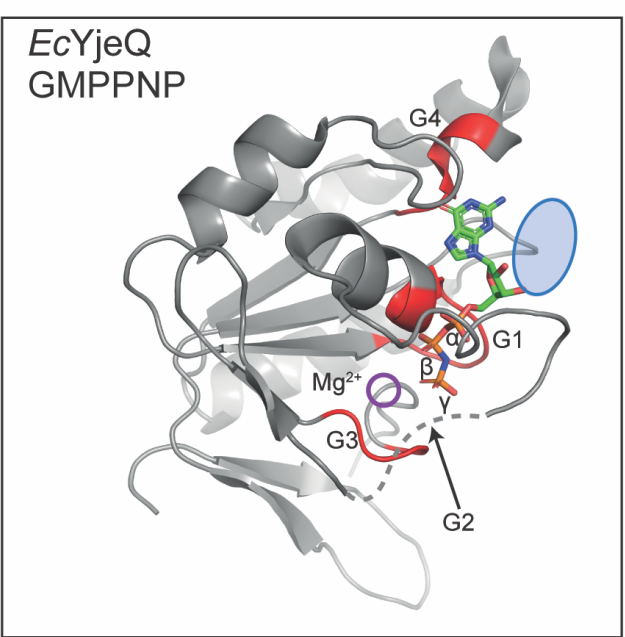

D

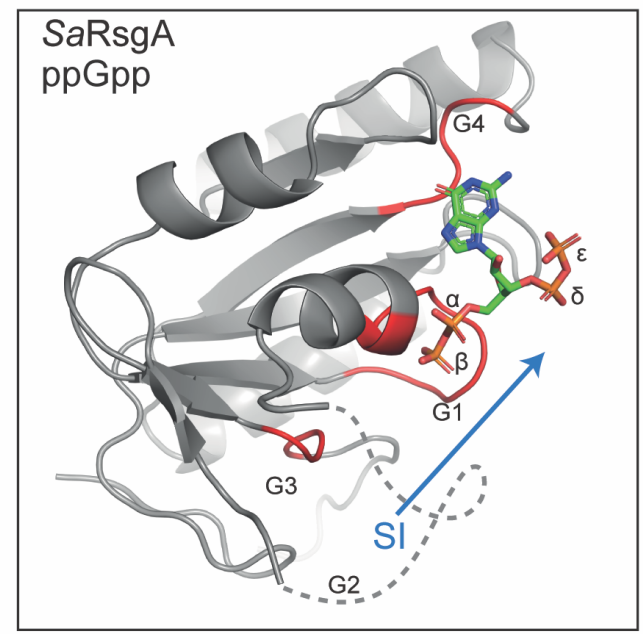

Figure 6. Comparison of the GTPase domains of RsgA and homologues in different nucleotide bound states. The G-domain conformation of A) Aquifex aeolicus RsgA (YjeQ) bound to GDP (PDB: 2YV5, chain A), B) Escherichia coli RsgA (YjeQ) bound to GMPPNP (PDB: 5UZ4, chain Z (47)), C) Staphylococcus aureus RsgA in the apo state (PDB: 6ZJO, chain A, this study) and D) Staphylococcus aureus RsgA bound to ppGpp (PDB: $6 Z \mathrm{HL}$, chain $\mathrm{A}$, this study). RsgA/YjeQ is shown as cartoon representations, coloured grey, with the G1, G2, G3 and G4 motifs coloured red where resolved. The hypothetical position of the switch I/G2 loop are represented by grey dashed lines as determined by comparison with the resolved region of the GDP-bound $\mathrm{YjeQ}$, and the bound nucleotides are coloured by atom as follows: carbon, green; nitrogen, blue; oxygen, red; phosphorous, orange. Rearrangements of the switch I/G2 loop to facilitate entry into the ON state are shown by blue arrows. The binding site of the $\mathrm{Mg}^{2+}$ ion in the GMPPNP-bound conformation (B) is indicated by a purple circle, and the position of the $\delta, \varepsilon$ - phosphate of ppGpp is indicated relative to bound GMPPNP by a blue oval in B. 
A

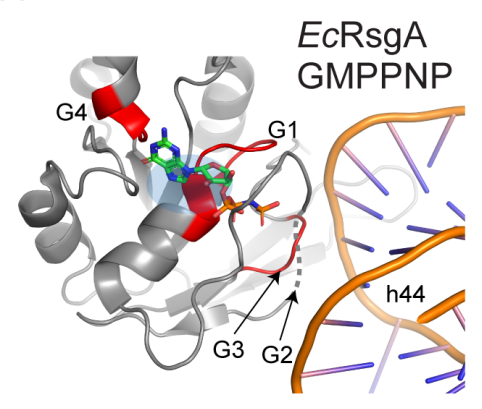

B

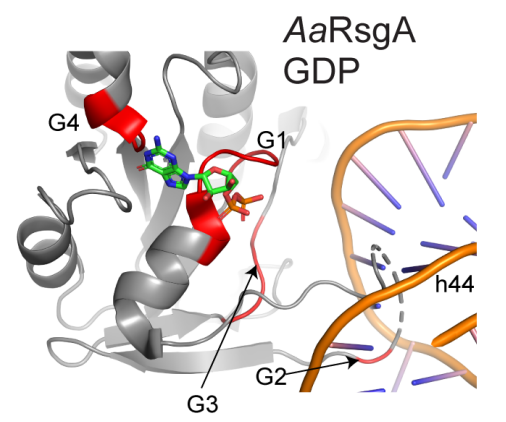

C

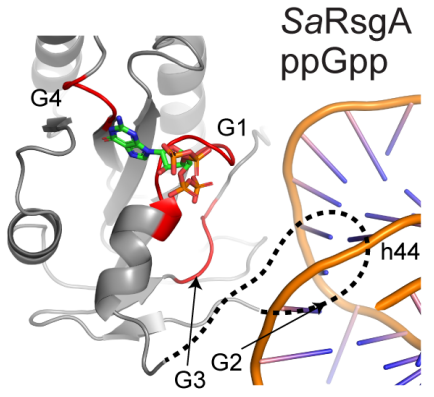

Figure 7. ppGpp-mediated inhibition of RA-GTPase association to ribosome subunits is facilitated by incorrect positioning of the switch I/G2 loop. A) E. coli RsgA (YjeQ) bound to GMPPNP (PDB: 5UZ4, chain Z) and chain A (16S rRNA) (47)) including a cropped view of the rRNA binding site on h44. For the full binding environment, see Figure 5. B, C) Docking of B) A. aeolicus RsgA (YjeQ) (PDB: 2YV5, chain A) bound to GDP and C) S. aureus RsgA bound to ppGpp (PDB: 6ZHL, chain A, this study) onto h44 of the $16 S$ rRNA from PDB:5UZ4 (chain A) using $C_{\alpha}$ alignment of the G-domains. The RsgA/YjeQ is shown as a cartoon representation coloured grey, with the G1, G2, G3 and G4 motifs coloured red where visible. The bound nucleotides are coloured by atom as follows: carbon, green; nitrogen, blue; oxygen, red; phosphorous, orange. Suggested conformations of unresolved regions of the switch I/G2 loop are represented by a black dashed line. 


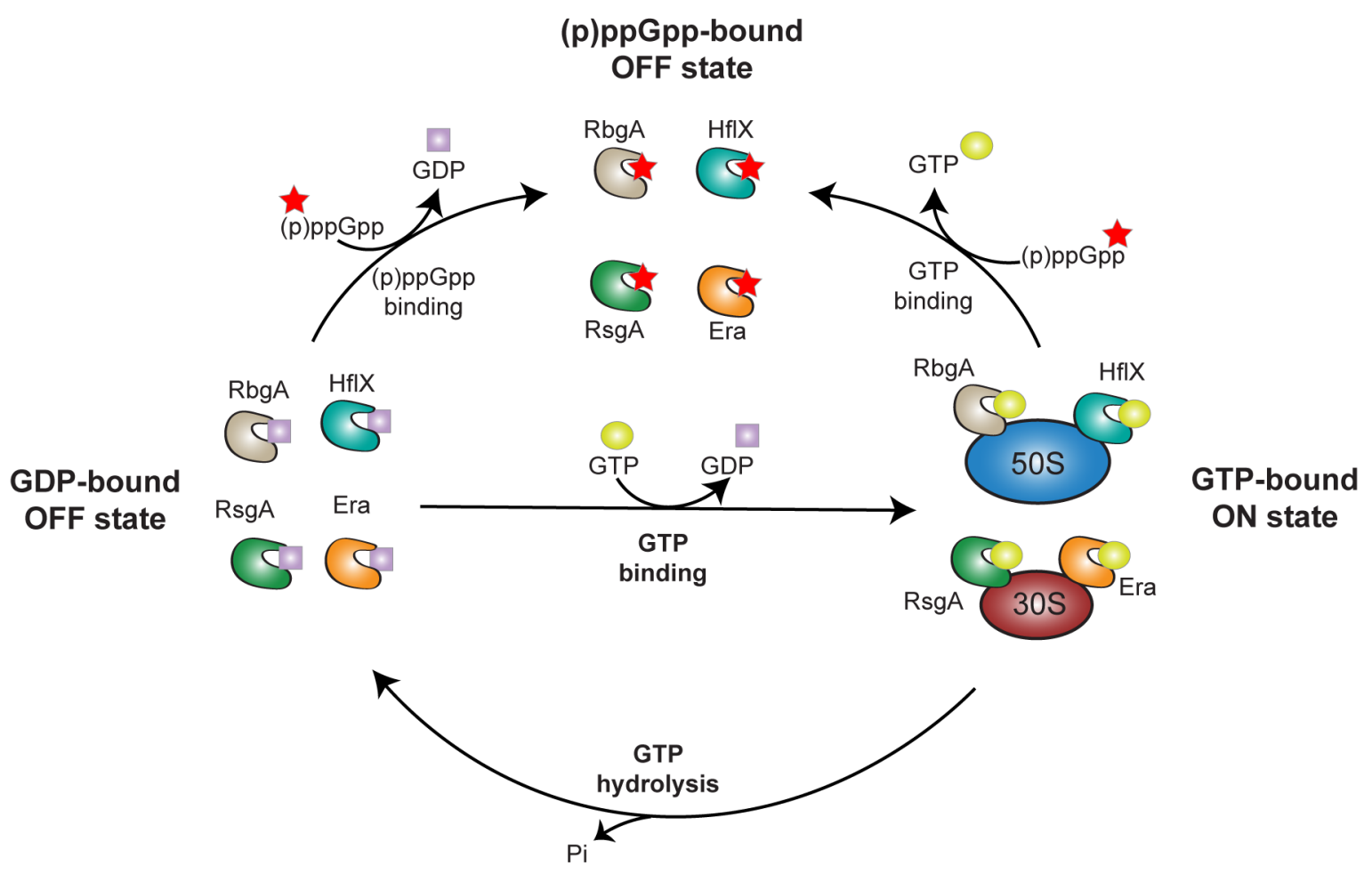

Figure 8. Model of the control of ribosome maturation by (p)ppGpp and RA-GTPases. Under proliferative conditions, GTP binds to RA-GTPases, enabling association to the immature ribosome subunits and subsequent maturation, at which point GTP is hydrolysed to GDP and the RA-GTPase dissociates from the ribosome. Under stringent conditions when cellular (p)ppGpp concentrations rise and GTP concentrations fall $(38,64)$, ( $p$ )ppGpp can outcompete GTP for RA-GTPase binding. This inhibits association of RA-GTPases to the ribosome subunits and negatively impacts ribosome biogenesis. 